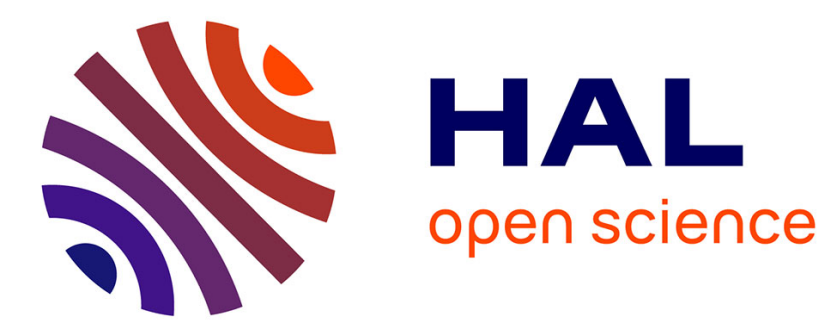

\title{
Multiple Timescales Analysis for 1:2 and 1:3 Resonant Hopf Bifurcations
}

\author{
Angelo Luongo, Achille Paolone, Angelo Di Egidio
}

\section{To cite this version:}

Angelo Luongo, Achille Paolone, Angelo Di Egidio. Multiple Timescales Analysis for 1:2 and 1:3 Resonant Hopf Bifurcations. Nonlinear Dynamics, 2003, 34 (3-4), pp.269-291. hal-00797874

\section{HAL Id: hal-00797874 \\ https://hal.science/hal-00797874}

Submitted on 7 Mar 2013

HAL is a multi-disciplinary open access archive for the deposit and dissemination of scientific research documents, whether they are published or not. The documents may come from teaching and research institutions in France or abroad, or from public or private research centers.
L'archive ouverte pluridisciplinaire HAL, est destinée au dépôt et à la diffusion de documents scientifiques de niveau recherche, publiés ou non, émanant des établissements d'enseignement et de recherche français ou étrangers, des laboratoires publics ou privés. 


\title{
Multiple Timescales Analysis for 1:2 and 1:3 Resonant Hopf Bifurcations
}

\author{
ANGELO LUONGO $^{1}$, ACHILLE PAOLONE$^{2}$, and ANGELO DI EGIDIO ${ }^{1}$ \\ ${ }^{1}$ Dipartimento di Ingegneria delle Strutture, delle Acque e del Terreno, Università di L'Aquila, \\ I-67040 Monteluco di Roio (L'Aquila), Italy; E-mail: \{luongo,ade\}@ing.univaq.it; ${ }^{2}$ Dipartimento di \\ Ingegneria Strutturale e Geotecnica, Università degli Studi di Roma 'La Sapienza', via Eudossiana 18, \\ I-00184 Roma, Italy; E-mail: achille.paolone@uniromal.it
}

\begin{abstract}
The postcritical behavior of a general $n$-dimensional system around a resonant double Hopf bifurcation is analyzed. Both cases in which the critical eigenvalues are in ratios of 1:2 and 1:3 are investigated. The Multiple Scale Method is employed to derive the bifurcation equations systematically in terms of the derivatives of the original vector field evaluated at the critical state. Expansions of the $n$-dimensional vector of state variables and of a three-dimensional vector of control parameters are performed in terms of a unique perturbation parameter $\varepsilon$, of the order of the amplitude of motion. However, while resonant terms only appear at the $\varepsilon^{3}$-order in the $1: 3$ case, they already arise at the $\varepsilon^{2}$-order in the 1:2 case. Thus, by truncating the analysis at the $\varepsilon^{3}$-order in both cases, first or second-order bifurcation equations are respectively drawn, the latter requiring resort to the reconstitution principle. A two-degrees-of-freedom system undergoing resonant double Hopf bifurcations is studied. The complete postcritical scenario is analyzed in terms of the three control parameters and the asymptotic results are compared with exact numerical integrations for both resonances. Branches of periodic as well as periodically modulated solutions are found and their stability analyzed.
\end{abstract}

Keywords: Multiple timescales, resonant, Hopf bifurcations, codimension-three, normal form bifurcation equations.

\section{Introduction}

The Multiple Timescale Method (MSM) has been widely used to analyze the dynamic response of weakly nonlinear mechanical systems in both free and forced oscillation regimes [1]. As in other reduction methods, the multiple timescale method transforms the evolution analysis of a multidimensional dynamic system into that of an equivalent dynamical problem of a dimension smaller than the original one and equal to its codimension [2]. The multiple timescale method often implies a smaller computational effort than other reduction methods [3]. Within bifurcation analysis, the main advantage of the method is the possibility of obtaining the reduced equations without describing the center manifold in advance or expressing the Jacobian matrix at the critical state in Jordan form. As a result, the bifurcation equations are obtained directly in standard normal form. Their coefficients are expressed in closed form in terms of the derivatives of the original vector field evaluated at the critical state, similarly to the theory of static bifurcation of conservative systems [4].

In the past, the method has been successfully applied by the authors to analyze codimension-two bifurcations of the nonresonant double-Hopf [5], Hopf-divergence [6], double-divergence types [7], and codimension-three bifurcations of the 1:1 resonant Hopf type [8]. In the last two cases, since the system Jacobian matrix is nilpotent at the bifurcation 
point, the classical method must be adapted. In particular, one-component generating solutions must be taken and, in addition, timescales with fractional powers of the perturbation parameter must be employed for the double divergence, similarly to the eigenvalue sensitivity analysis of nilpotent matrices $[9,10]$.

In $[11,12]$ the MSM has been used to analyze the free vibrations of weakly nonlinear twodegrees-of-freedom oscillators for the nonresonant and 1:3 internal resonance cases. However, the approach followed therein is somewhat different from the present method. The system is almost Hamiltonian, whereas here, general nonconservative systems are considered. This entails critical eigenvectors that are not simply real but are, in general, complex. In addition, in these cases, the method has been employed not as a reduction method but as a tool to simplify the nonlinear equations of motion. In other words, it has been adopted in a manner similar to that of the normal form method usually employed to transform the bifurcation equations furnished by the center manifold reduction to standard normal form [13].

In this paper, the multiple timescale method is applied to analyze a general dynamic system undergoing a resonant double-Hopf bifurcation of codimension three. Two different resonances are studied, namely the 1:2 and 1:3 type. The relevant complex bifurcation equations are recast in real form by performing a nonstandard change of variables based on the definition of generalized amplitudes of motion [14]. The procedure is applied to investigate the post-critical scenario of a simple mechanical system subjected to aerodynamic excitation.

\section{Position of the Problem}

An autonomous dynamical system is considered, governed by the following equation of motion:

$$
\dot{\mathbf{x}}=\mathbf{F}(\mathbf{x}, \boldsymbol{\mu}),
$$

where $\mathbf{x} \in \mathbb{R}^{n}$ is the state vector and $\boldsymbol{\mu}$ is the control parameter vector. According to the bifurcation theory, a number of parameters not lower than the codimension of the bifurcation must be taken into account, in order that the bifurcation is structurally stable. A resonant double-Hopf bifurcation is a (linear) codimension-three bifurcation, since three degenerateness conditions among two eigenvalues $\lambda_{k}$ hold at it, namely $\operatorname{Re} \lambda_{1}=\operatorname{Re} \lambda_{2}=0$, $\operatorname{Im} \lambda_{2}=\beta \operatorname{Im} \lambda_{1}$ with $\beta=2,3$, in the $1: 2$ and 1:3 resonances, respectively. Therefore, $\boldsymbol{\mu}=\{\mu \nu \eta\}^{T} \in \mathbb{R}^{3}$ is taken.

By hypothesis, Equations (1) admit the trivial equilibrium solution $\mathbf{x}=\mathbf{0}$ for any value of $\boldsymbol{\mu}$. The point $O \equiv(\mathbf{x}=\mathbf{0}, \boldsymbol{\mu}=\mathbf{0})$ is assumed to be a bifurcation point. The following hypotheses are assumed to hold for the Jacobian matrix at $O$

$$
\mathbf{F}_{\mathbf{x}}^{0}:=\left.\frac{\partial \mathbf{F}(\mathbf{x}, \boldsymbol{\mu})}{\partial \mathbf{x}}\right|_{\mathbf{x}=\mathbf{0}, \boldsymbol{\mu}=\mathbf{0}}
$$

(H.1) The Jacobian matrix $\mathbf{F}_{\mathbf{x}}^{0}$ has two pairs of purely imaginary eigenvalues $\lambda_{1,3}=$ $\pm i \omega_{10}, \quad \lambda_{2,4}= \pm i \omega_{20}$. In addition, the frequencies $\omega_{10}$ and $\omega_{20}$ are assumed to be resonant, i.e. $\omega_{20}=\beta \omega_{10}$, with $\beta=2,3$. The associated right $\mathbf{u}_{j}$ and left $\mathbf{v}_{j}(j=1,2)$ eigenvectors are solutions of the following algebraic equations;

$$
\left(\mathbf{F}_{\mathbf{x}}^{0}-i \mathbf{E} \omega_{j 0}\right) \mathbf{u}_{j}=\mathbf{0}, \quad\left(\mathbf{F}_{\mathbf{x}}^{0^{T}}+i \mathbf{E} \omega_{j 0}\right) \mathbf{v}_{j}=\mathbf{0},
$$


where $\mathbf{E}$ is the identity matrix, $\mathbf{u}_{3}=\overline{\mathbf{u}}_{1}, \mathbf{u}_{4}=\overline{\mathbf{u}}_{2}, \mathbf{v}_{3}=\overline{\mathbf{v}}_{1}$ and $\mathbf{v}_{4}=\overline{\mathbf{v}}_{2}$. Right and left eigenvectors are orthonormal, i.e. $\mathbf{v}_{i}^{H} \mathbf{u}_{j}=\delta_{i j}$, where $H$ denotes the transpose conjugate and $\delta_{i j}$ is the Kronecker delta.

(H.2) At bifurcation, all the remaining eigenvalues $\lambda_{h}, h \geq 5$, lie on the left side of the complex plane with negative real part of at least $O(1)$.

(H.3) The critical eigenvalues

$$
\begin{aligned}
& \lambda_{1,3}=\alpha_{1}(\mu, v, \eta) \pm i \omega_{1}(\mu, v, \eta), \\
& \lambda_{2,4}=\alpha_{2}(\mu, v, \eta) \pm i \omega_{2}(\mu, v, \eta),
\end{aligned}
$$

in which $\alpha_{j}(0,0,0)=0,(j=1,2)$ and $\omega_{2}(0,0,0)=\beta \omega_{1}(0,0,0),(\beta=2,3)$, satisfy the following transversality conditions at the bifurcation point

$$
\operatorname{det}\left[\begin{array}{ccc}
\alpha_{1 \mu}^{0} & \alpha_{1 v}^{0} & \alpha_{1 \eta}^{0} \\
\alpha_{2 \mu}^{0} & \alpha_{2 v}^{0} & \alpha_{2 \eta}^{0}
\end{array}\right] \neq 0
$$

where

$$
\alpha_{j \mu}^{0}:=\left.\frac{\partial \alpha_{j}}{\partial \mu}\right|_{\substack{\mu=0 \\ \nu=0 \\ \eta=0}}, \quad \alpha_{j \nu}^{0}:=\left.\frac{\partial \alpha_{j}}{\partial \nu}\right|_{\substack{\mu=0 \\ \nu=0 \\ \eta=0}}, \quad \alpha_{j \eta}^{0}:=\left.\frac{\partial \alpha_{j}}{\partial \eta}\right|_{\substack{\mu=0 \\ \nu=0 \\ \eta=0}}, \quad(j=1,2) .
$$

In the parameter space $(\mu, v, \eta)$, the critical surfaces $\alpha_{j}(\mu, v, \eta)=0(j=1,2)$, bound the regions of linear stability of the trivial solution, whereas the surface $\omega_{2}(\mu, v, \eta)=$ $\beta \omega_{1}(\mu, v, \eta)$ is the locus where the resonance among the critical eigenvalues occurs. By perturbing the critical parameters along the direction tangential to the curve $\alpha_{1}=$ $\alpha_{2}=0$, nearly-resonant Hopf bifurcations are encountered, for which $\omega_{2}-\beta \omega_{1} \approx 0$. Such a perturbation plays, therefore, the role of detuning parameters among the critical frequencies. Perturbations normal to that directions govern instead the splitting among the two Hopf bifurcations, as it occurs for nonresonant bifurcations [2]. According to (5), the critical surfaces have distinct normal at the intersection point $O$, where the codimension-3 bifurcation takes place, so that no direction in the $(\mu, v, \eta)$-space exists along which the critical state persists.

In the following, the multiple scale method is applied to analyze the postcritical behavior around the bifurcation point.

\section{The Multiple Scale Method}

According to the MSM, a monoparametric family of solutions of the type

$$
\mathbf{x}=\mathbf{x}\left(\varepsilon, t_{k}, \ldots\right), \quad \boldsymbol{\mu}=\boldsymbol{\mu}(\varepsilon)
$$

is sought for Equation (1), where $t_{k}=\varepsilon^{k} t(k=0,1, \ldots)$ for the $1: 2$ case and $(k=0,2,4, \ldots)$ for the 1:3 case respectively, are independent temporal scales and $\varepsilon \ll 1$ is a perturbation parameter which vanishes at the bifurcation point. By using the chain rule $\mathrm{d} / \mathrm{d} t=\sum_{k=0}^{\infty} \varepsilon^{k} d_{k}$ with $d_{k}:=\partial / \partial t_{k}$. Under hypotheses of regularity, the state vector is expanded in series of $\varepsilon$ as

$$
\mathbf{x}=\sum_{h=1}^{\infty} \varepsilon^{h} \mathbf{x}_{h}
$$


while the vector parameter is ordered as

$$
\boldsymbol{\mu}=\varepsilon^{\gamma} \widehat{\boldsymbol{\mu}}
$$

with $\widehat{\mu}=O(1)$ and the integer $\gamma$ properly selected in such a way that $\widehat{\mu}$ appears in the perturbation scheme at the same level as the first resonant terms.

In the following, the 1:2 and 1:3 cases are examined separately.

\subsection{1:2 RESONANCE}

By substituting Equations (8) and (9), in (1), choosing $\gamma=1$, expanding $\mathbf{F}$ as well and equating separately to zero terms with the same powers of $\varepsilon$, the following perturbative equations are obtained

$$
\begin{aligned}
\left(d_{0}-\mathbf{F}_{\mathbf{x}}^{0}\right) \mathbf{x}_{1}= & \mathbf{0} \\
\left(d_{0}-\mathbf{F}_{\mathbf{x}}^{0}\right) \mathbf{x}_{2}= & -d_{1} \mathbf{x}_{1}+\mathbf{F}_{\mathbf{x} \mu}^{0} \mathbf{x}_{1} \widehat{\boldsymbol{\mu}}+\frac{1}{2} \mathbf{F}_{\mathbf{x x}}^{0} \mathbf{x}_{1}^{2} \\
\left(d_{0}-\mathbf{F}_{\mathbf{x}}^{0}\right) \mathbf{x}_{3}= & -d_{1} \mathbf{x}_{2}-d_{2} \mathbf{x}_{1}+\mathbf{F}_{\mathbf{x} \mu}^{0} \mathbf{x}_{2} \widehat{\boldsymbol{\mu}}+\mathbf{F}_{\mathbf{x} \mu \mu}^{0} \mathbf{x}_{1} \widehat{\boldsymbol{\mu}}^{2}+\mathbf{F}_{\mathbf{x} x \mu}^{0} \mathbf{x}_{1}^{2} \widehat{\boldsymbol{\mu}} \\
& +\mathbf{F}_{\mathbf{x} \mathbf{x}}^{0} \mathbf{x}_{1} \mathbf{x}_{2}+\frac{1}{6} \mathbf{F}_{\mathbf{x x x}}^{0} \mathbf{x}_{1}^{3},
\end{aligned}
$$

where $\mathbf{F}_{\mathbf{x} \mu}^{0}:=\partial^{2} \mathbf{F}(\mathbf{0}, \mathbf{0}) / \partial \mathbf{x} \partial \boldsymbol{\mu}$ and similarly for higher-order derivatives.

The nondecaying solution of Equation $\left(10_{1}\right)$ is

$$
\mathbf{x}_{1}=A_{1}\left(t_{1}, t_{2}, \ldots\right) \mathbf{u}_{1} \mathrm{e}^{i \omega_{10} t_{0}}+A_{2}\left(t_{1}, t_{2}, \ldots\right) \mathbf{u}_{2} \mathrm{e}^{i \omega_{20} t_{0}}+\text { c.c. }
$$

where $A_{j}(j=1,2)$ are complex constants, $\mathbf{u}_{j}$ are the right eigenvectors of $\mathbf{F}_{\mathbf{x}}^{0}$ associated with the eigenvalues $i \omega_{j 0}$ (Equation $\left.\left(3_{1}\right)\right)$ and c.c. stands for the complex conjugate of the preceding terms. With Equation $(11)$ and $\left(10_{2}\right)$ reads

$$
\begin{aligned}
\left(d_{0}-\mathbf{F}_{\mathbf{x}}^{0}\right) \mathbf{x}_{2}= & \left(-d_{1} A_{1} \mathbf{u}_{1}+A_{1} \mathbf{F}_{\mathbf{x} \mu}^{0} \mathbf{u}_{1} \widehat{\boldsymbol{\mu}}\right) \mathrm{e}^{i \omega_{10} t_{0}} \\
& +\left(-d_{1} A_{2} \mathbf{u}_{2}+A_{2} \mathbf{F}_{\mathbf{x} \mu}^{0} \mathbf{u}_{2} \widehat{\boldsymbol{\mu}}\right) \mathrm{e}^{i \omega_{20} t_{0}} \\
& +\frac{1}{2} A_{1}^{2} \mathbf{F}_{\mathbf{x} \mathbf{x}}^{0} \mathbf{u}_{1}^{2} \mathrm{e}^{i 2 \omega_{10} t_{0}}+\frac{1}{2} A_{2}^{2} \mathbf{F}_{\mathbf{x} \mathbf{x}}^{0} \mathbf{u}_{2}^{2} \mathrm{e}^{i 2 \omega_{20} t_{0}} \\
& +\frac{1}{2} A_{1} \bar{A}_{1} \mathbf{F}_{\mathbf{x} \mathbf{x}}^{0} \mathbf{u}_{1} \overline{\mathbf{u}}_{1}+\frac{1}{2} A_{2} \bar{A}_{2} \mathbf{F}_{\mathbf{x x}}^{0} \mathbf{u}_{2} \overline{\mathbf{u}}_{2} \\
& +A_{1} A_{2} \mathbf{F}_{\mathbf{x x}}^{0} \mathbf{u}_{1} \mathbf{u}_{2} \mathrm{e}^{i\left(\omega_{10}+\omega_{20}\right) t_{0}}+\bar{A}_{1} A_{2} \mathbf{F}_{\mathbf{x} \mathbf{x}}^{0} \overline{\mathbf{u}}_{1} \mathbf{u}_{2} \mathrm{e}^{i\left(\omega_{20}-\omega_{10}\right) t_{0}}+\text { c.c. }
\end{aligned}
$$

This equation contains terms that would lead to secular terms; to eliminate them, the orthogonality of the coefficients of the resonant terms to the left eigenvectors $\mathbf{v}_{j}$ has to be imposed, leading to

$$
\begin{aligned}
& d_{1} A_{1}=\mathbf{C}_{1 \mu} \widehat{\boldsymbol{\mu}} A_{1}+C_{\overline{1} 2} \bar{A}_{1} A_{2} \\
& d_{1} A_{2}=\mathbf{C}_{2 \mu} \widehat{\boldsymbol{\mu}} A_{2}+C_{11} A_{1}^{2},
\end{aligned}
$$


where the resonance condition $\omega_{20}=2 \omega_{10}$ has been taken into account and the row vector $\mathbf{C}_{j \mu} \in \mathbb{C}^{3}(j=1,2)$ and the coefficients $C_{i j} \in \mathbb{C}$ are given in Appendix A. Equation(12) then furnishes

$$
\begin{aligned}
\mathbf{x}_{2}= & \left(A_{1} \mathbf{Z}_{1 \mu} \widehat{\boldsymbol{\mu}}+\bar{A}_{1} A_{2} \mathbf{Z}_{\overline{1} 2}\right) \mathrm{e}^{i \omega_{10} t_{0}}+\left(A_{2} \mathbf{Z}_{2 \mu} \widehat{\boldsymbol{\mu}}+A_{1}^{2} \mathbf{Z}_{11}\right) \mathrm{e}^{i \omega_{20} t_{0}} \\
& +A_{2}^{2} \mathbf{z}_{22} \mathrm{e}^{i 2 \omega_{20} t_{0}}+A_{1} A_{2} \mathbf{z}_{12} \mathrm{e}^{i\left(\omega_{10}+\omega_{20}\right) t_{0}}+A_{1} \bar{A}_{1} \mathbf{z}_{1 \overline{1}}+A_{2} \bar{A}_{2} \mathbf{z}_{2 \overline{2}}+\text { c.c. },
\end{aligned}
$$

where $\mathbf{z}_{i j}, \mathbf{Z}_{i j} \in \mathbb{C}^{n}$ and $\mathbf{Z}_{j \mu} \in \mathbb{C}^{n} \times \mathbb{C}^{3}$ are particular solutions of the linear algebraic equations reported in Appendix A. By substituting Equations $(11,14)$ into $\left(10_{3}\right)$ and applying the usual procedures, the following solvability conditions are determined

$$
\begin{aligned}
& d_{2} A_{1}=\mathbf{C}_{1 \mu \mu} \widehat{\boldsymbol{\mu}}^{2} A_{1}+\mathbf{C}_{\overline{1} 2 \mu} \widehat{\boldsymbol{\mu}} \bar{A}_{1} A_{2}+C_{12 \overline{2}} A_{1} A_{2} \bar{A}_{2}+C_{11 \overline{1}} A_{1}^{2} \bar{A}_{1} \\
& d_{2} A_{2}=\mathbf{C}_{2 \mu \mu} \widehat{\boldsymbol{\mu}}^{2} A_{2}+\mathbf{C}_{11 \mu} \widehat{\boldsymbol{\mu}} A_{1}^{2}+C_{1 \overline{1} 2} A_{1} \bar{A}_{1} A_{2}+C_{22 \overline{2}} A_{2}^{2} \bar{A}_{2},
\end{aligned}
$$

where $\mathbf{C}_{i j \mu} \in \mathbb{C}^{3}, \mathbf{C}_{i \mu \mu} \in \mathbb{C}^{3} \times \mathbb{C}^{3}$ and $C_{i j h} \in \mathbb{C}$ are given in Appendix A.

By returning to the actual time $t$, Equations $(13,15)$ can be combined in a single equation (reconstitution method [2]). By taking into account that

$$
\frac{\mathrm{d} A_{j}}{\mathrm{~d} t}=\varepsilon d_{1} A_{j}+\varepsilon^{2} d_{2} A_{j}+O\left(\varepsilon^{3}\right), \quad j=1,2
$$

and using the solvability conditions (Equations $(13,15)$ ), the following bifurcation equations are obtained

$$
\begin{aligned}
& \dot{A}_{1}=\left(\mathbf{C}_{1 \mu} \widehat{\boldsymbol{\mu}}+\mathbf{C}_{1 \mu \mu} \widehat{\boldsymbol{\mu}}^{2}\right) A_{1}+\left(\mathbf{C}_{\overline{12}}+\mathbf{C}_{\overline{12} 2} \widehat{\boldsymbol{\mu}}\right) \bar{A}_{1} A_{2}+C_{12 \overline{2}} A_{1} A_{2} \bar{A}_{2}+C_{11 \overline{1}} A_{1}^{2} \bar{A}_{1} \\
& \dot{A}_{2}=\left(\mathbf{C}_{1 \mu} \widehat{\boldsymbol{\mu}}+\mathbf{C}_{1 \mu \mu} \widehat{\boldsymbol{\mu}}^{2}\right) A_{2}+\left(C_{11}+\mathbf{C}_{11 \mu} \widehat{\boldsymbol{\mu}}\right) A_{1}^{2}+C_{1 \overline{1} 2} A_{1} \bar{A}_{1} A_{2}+C_{22 \overline{2}} A_{2}^{2} \bar{A}_{2},
\end{aligned}
$$

where the parameter $\varepsilon$ has been reabsorbed according to Equation (16) and to the rules $\varepsilon A_{j} \rightarrow$ $A_{j}, \varepsilon \mathrm{d} / \mathrm{d} \rightarrow \mathrm{d} / \mathrm{d} t$.

To put the bifurcation equations in real form, e.g. useful to employ automatic tools as AUTO [15], it is convenient to adopt a mixed (polar and Cartesian) form for the complex amplitudes, namely [14]

$$
A_{1}=\frac{1}{2} a \mathrm{e}^{i \theta}, \quad A_{2}=\frac{1}{2}(u+i v) \mathrm{e}^{i 2 \theta} .
$$

Substituting Equations (18) into (17) and separating real and imaginary parts yealds

$$
\begin{aligned}
& \dot{a}=f(a, u, v), \\
& \dot{u}=g(a, u, v, \dot{\theta}), \\
& \dot{v}=h(a, u, v, \dot{\theta}), \\
& a \dot{\theta}=a \psi(a, u, v),
\end{aligned}
$$

where $f, g, h$ and $\psi$ are cubic polynomials in the variables $(a, u, v)$. From Equation $\left(19_{4}\right)$ $\dot{\theta}$ can be expressed as a function of $a, u, v$ and substituted in $\left(19_{2}\right)$ and $\left(19_{3}\right)$. To perform this operation, Equation (194) must be divided by $a$, so that the trivial solution $a=0$ could be lost. However, it is possible to check that such a division is allowed, since the trivial solution is still admitted by the reduced system and the stability properties of the system are not altered 
(Appendix C). Therefore $\dot{\theta}$ can actually be eliminated in the first three equations. It should be noticed that the factorization in $a$ of Equation $\left(19_{4}\right)$ is a consequence of the factorization in $A_{1}$ of Equation $\left(17_{1}\right)$ and of its polar representation $\left(18_{1}\right)$. It follows that the amplitude modulation equations $\left(19_{1,2,3}\right)$ (equal in number to the linear codimension of the problem) are uncoupled from the phase modulation $\theta$.

\subsection{1:3 RESONANCE}

By ordering the control parameters as $\boldsymbol{\mu}=\varepsilon^{2} \hat{\boldsymbol{\mu}}$, i.e. by choosing $\gamma=2$ in Equation (9), and applying the same procedure as in the previous case, the following perturbative equations are obtained

$$
\begin{aligned}
& \left(d_{0}-\mathbf{F}_{\mathbf{x}}^{0}\right) \mathbf{x}_{1}=\mathbf{0} \\
& \left(d_{0}-\mathbf{F}_{\mathbf{x}}^{0}\right) \mathbf{x}_{2}=\frac{1}{2} \mathbf{F}_{\mathbf{x} \mathbf{x}}^{0} \mathbf{x}_{1}^{2} \\
& \left(d_{0}-\mathbf{F}_{\mathbf{x}}^{0}\right) \mathbf{x}_{3}=-d_{2} \mathbf{x}_{1}+\mathbf{F}_{\mathbf{x} \mu}^{0} \mathbf{x}_{1} \hat{\boldsymbol{\mu}}+\mathbf{F}_{\mathbf{x x}}^{0} \mathbf{x}_{1} \mathbf{x}_{2}+\frac{1}{6} \mathbf{F}_{\mathbf{x x x}}^{0} \mathbf{x}_{1}^{3} .
\end{aligned}
$$

The generating solution of Equation $\left(20_{1}\right)$ is still given by Equation (11). By substituting it into Equation $\left(20_{2}\right)$, no resonant terms appear. Solving it yields

$$
\begin{aligned}
\mathbf{x}_{2}= & A_{1}^{2} \mathbf{z}_{11} \mathrm{e}^{i 2 \omega_{10} t_{0}}+A_{2}^{2} \mathbf{z}_{22} \mathrm{e}^{i 2 \omega_{20} t_{0}}+A_{1} \bar{A}_{1} \mathbf{z}_{1 \overline{1}}+A_{2} \bar{A}_{2} \mathbf{z}_{2 \overline{2}} \\
& +A_{1} A_{2} \mathbf{z}_{12} \mathrm{e}^{i\left(\omega_{10}+\omega_{20}\right) t_{0}}+A_{1} \bar{A}_{2} \mathbf{z}_{1 \overline{2}} \mathrm{e}^{i\left(\omega_{10}-\omega_{20}\right) t_{0}}+\text { c.c. },
\end{aligned}
$$

where the $\mathbf{z}_{r s}$ 's and $\mathbf{z}_{r \bar{s}}$ 's $(r, s=1,2) \in \mathbb{C}^{n}$ are particular solutions of the linear nonsingular algebraic equations (27) of Appendix A.

By going further to a higher order and applying the same procedure as in the previous case, the following bifurcation equations are obtained

$$
\begin{aligned}
& \dot{A}_{1}=\mathbf{C}_{1 \mu} \hat{\mu} A_{1}+C_{11 \overline{1}} A_{1}^{2} \bar{A}_{1}+C_{\overline{1} \overline{1} 2} \bar{A}_{1}^{2} A_{2}+C_{12 \overline{2}} A_{1} A_{2} \bar{A}_{2} \\
& \dot{A}_{2}=\mathbf{C}_{2 \mu} \hat{\mu} A_{2}+C_{111} A_{1}^{3}+C_{1 \overline{1} 2} A_{1} \bar{A}_{1} A_{2}+C_{22 \overline{2}} A_{2}^{2} \bar{A}_{2},
\end{aligned}
$$

where the parameter $\varepsilon$ has been reabsorbed by taking into account that

$$
\frac{\mathrm{d} A_{j}}{\mathrm{~d} t}=\varepsilon^{2} d_{2} A_{j}+O\left(\varepsilon^{4}\right)
$$

and the rules $\varepsilon A_{j} \rightarrow A_{j}, \varepsilon^{2}(\mathrm{~d} / \mathrm{d} t) \rightarrow \mathrm{d} / \mathrm{d} t$. The expression of the vector rows $\mathbf{C}_{j \mu} \in \mathbb{C}^{3}$ and the coefficients $C_{i j h} \in \mathbb{C}$ are reported in Appendix B.

The standard normal form of the bifurcation equations is again obtained by using a mixedform representation for the amplitudes

$$
A_{1}=\frac{1}{2} a \mathrm{e}^{i \theta}, \quad A_{2}=\frac{1}{2}(u+i v) \mathrm{e}^{i 3 \theta} .
$$

By separating the real and imaginary parts in Equations (22), the generalized amplitudes and phase modulation equations are drawn. They possess the same structure as in the 1:2 resonance case (Equations (19)), i.e. the generalized amplitude equations are uncoupled from the phase equation. 

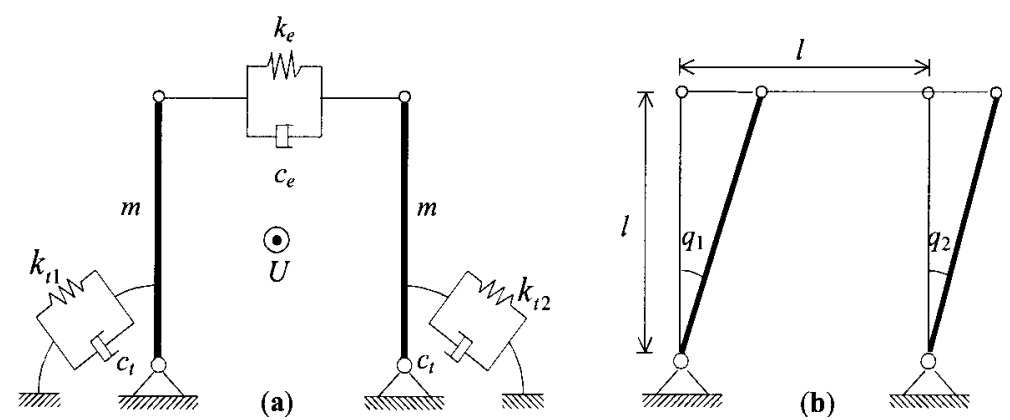

Figure 1. (a) System of two bars under aerodynamic exitation; (b) Lagrangian parameters.

It can be checked that bifurcation equations, when they are expressed in polar coordinates $A_{k}=a_{k} \mathrm{e}^{i \theta_{k}}$ are invariant with respect to the change $a_{k} \rightarrow-a_{k}, \theta_{k}=\theta_{k}+\pi(i=1,2)$. Therefore, solitions can be sought only in the positive amplitude half-plane.

\section{Sample Structure}

\subsection{EquATIONS OF Motion AND Bifurcation ANALYSis}

In this section, the structure of Figure 1 is analyzed. It consists of two vertical rigid bars of length $l$, constrained by two different linear visco-elastic hinges and joined at their ends by a nonlinear visco-elastic control device, producing a restoring force $f=f(e)$, where $e$ is the strain, that can have either positive or negative linear stiffness. The structure is loaded by a fluid flow of uniform velocity $U$ orthogonal to the plane of the motion. If the constitutive nonlinearities of the extensional spring are neglected, the structure is reduced to that studied in [5]. By assuming the rotations $q_{i}(i=1,2)$ as Lagrangian parameters (Figure 1b), applying the quasi-static theory to express the aerodynamic forces [16], the nondimensional equations of motion, expanded up to third order, are of the kind

$$
\dot{\mathbf{x}}=\mathbf{A}(\hat{\mu}, \hat{v}, \hat{\eta}) \mathbf{x}+\mathbf{b}(\mathbf{x} ; \hat{\mu}, \hat{v}, \hat{\eta}),
$$

where the dot denotes differentiation with respect to the dimensionless temporal scale $\tau$; $\mathbf{x}=\left(x_{1}, x_{2}, x_{3}, x_{4}\right)^{T}=\left(q_{1}, \dot{q}_{1}, q_{2}, \dot{q}_{2}\right)^{T}$ is the state vector; $\mathbf{A}$ is the Jacobian matrix at $O$ and $\mathbf{b}(\mathbf{x})=\left(0, b_{1}(\mathbf{x}), 0, b_{2}(\mathbf{x})\right)^{T}$ is the vector collecting quadratic and cubic nonlinearities, In Equations $(25)(\hat{\mu}, \hat{v}, \hat{\eta}):=(\mu, v, \eta)-\left(\mu_{0}, v_{0}, \eta_{0}\right)$ are the increment of the control parameters $\mu$ (nondimensional wind velocity), $v$ (nondimensional linear stiffness of the extensional spring) and $\eta$ (linear torsional stiffness ratio), while $\mu_{0}, v_{0}, \eta_{0}$ are the values of the control parameters at the critical state. In the following, the hat has been omitted for simplicity (see Appendix D for more details).

\subsection{1:3 RESONANCE}

The linear stability diagram in the three-dimensional control parameter space was first constructed. It was obtained by analyzing the eigenvalues of the linearized part of both the bifurcation equations and the original equations of motion (25) and the results were then compared. Of course, since the fast dynamics are filtered in the bifurcation equations, the two sets of eigenvalues (approximatively) differ by the imaginary quantities $i \omega$ and $3 i \omega$. 
(a)

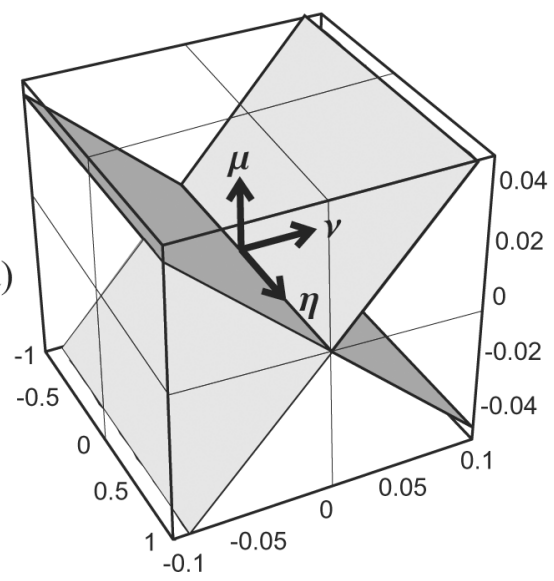

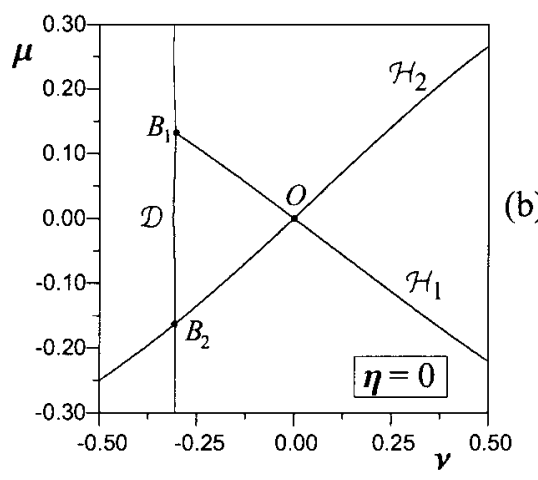

(b)
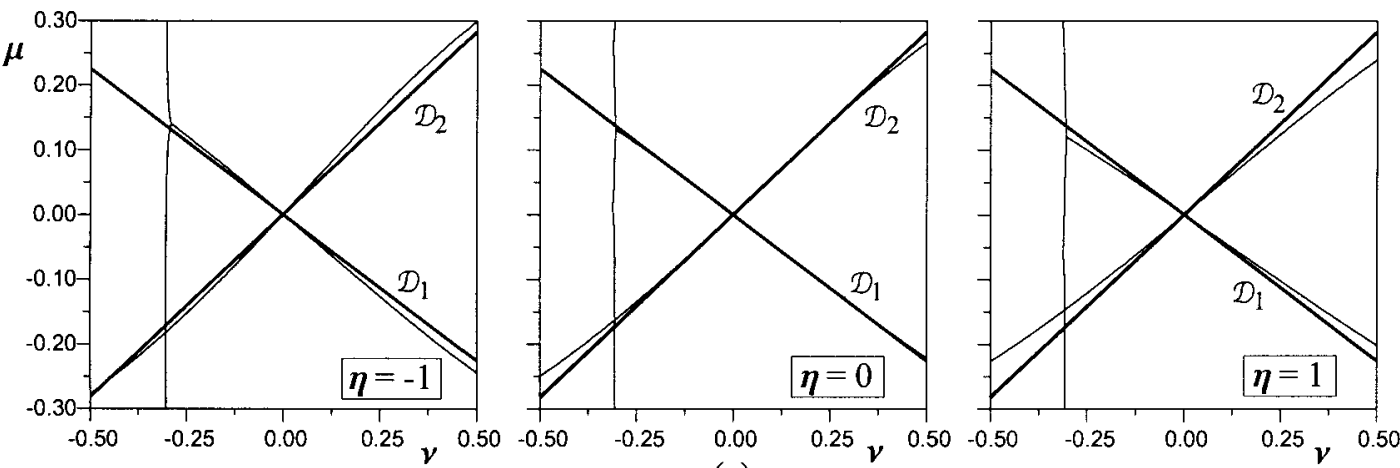

(c)

Figure 2. Linear stability diagrams for 1:3 resonance: (a) three-dimensional view of the asymptotic boundaries; (b) exact boundaries; (c) comparison between asymptotic (heavy lines) and exact (thin lines) boundaries.

Therefore, Hopf bifurcations for the state variables $\mathbf{x}$ appear as divergence bifurcations for the amplitude variables $(a, u, v)$. The obtained critical boundaries have been represented in Figure 2. A three-dimensional view of the asymptotic boundaries is given in Figure 2a. The planes both contain the $\eta$-axis, since, at the leading order, the parameter $\eta$ only affects the resonance conditions. A section of the exact boundaries at $\eta=0$ is shown in Figure $2 b$. Two Hopf boundaries $\mathscr{H}_{1}$ and $\mathscr{H}_{2}$ arise from the bifurcation point $O$, while a third divergence boundary $\mathscr{D}$ is found in the neighborhood of the origin. Consequently, a double-zero and a Hopf-divergence bifurcation occur at the bifurcation points $B_{1}$ and $B_{2}$, respectively. When $\eta$ is varied, the exact boundaries (thin lines) change according to Figure $2 c$, where a clockwise rotation is displayed, while the asymptotic boundaries (heavy lines), do not change. According to the asymptotic analysis, the boundaries have been labeled as divergence boundaries $\mathscr{D}_{1}$ and $\mathscr{D}_{2}$. A bimodal equilibrium solution $\mathcal{E}_{1}:=\left(a_{1}, a_{2}\right)$ (i.e., a periodic solution $\mathbf{x}$ of frequency close to the lower frequency $\left.\omega_{10}\right)$ bifurcates from $\mathscr{D}_{1}$, while a monomodal equilibrium solution $\mathcal{E}_{2}:=\left(0, a_{2}\right)$ (i.e., a periodic solution $\mathbf{x}$ of frequency close to the higher frequency $\omega_{20}$ ) bifurcates from $\mathscr{D}_{2}$, with $a_{1}:=\left|A_{1}\right|=a, a_{2}:=\left|A_{2}\right|=\left(u^{2}+v^{2}\right)^{1 / 2}$ (Equation (24)). The third boundary is lost in the bifurcation equation, so that the investigation is limited to a smaller region around $O$. 

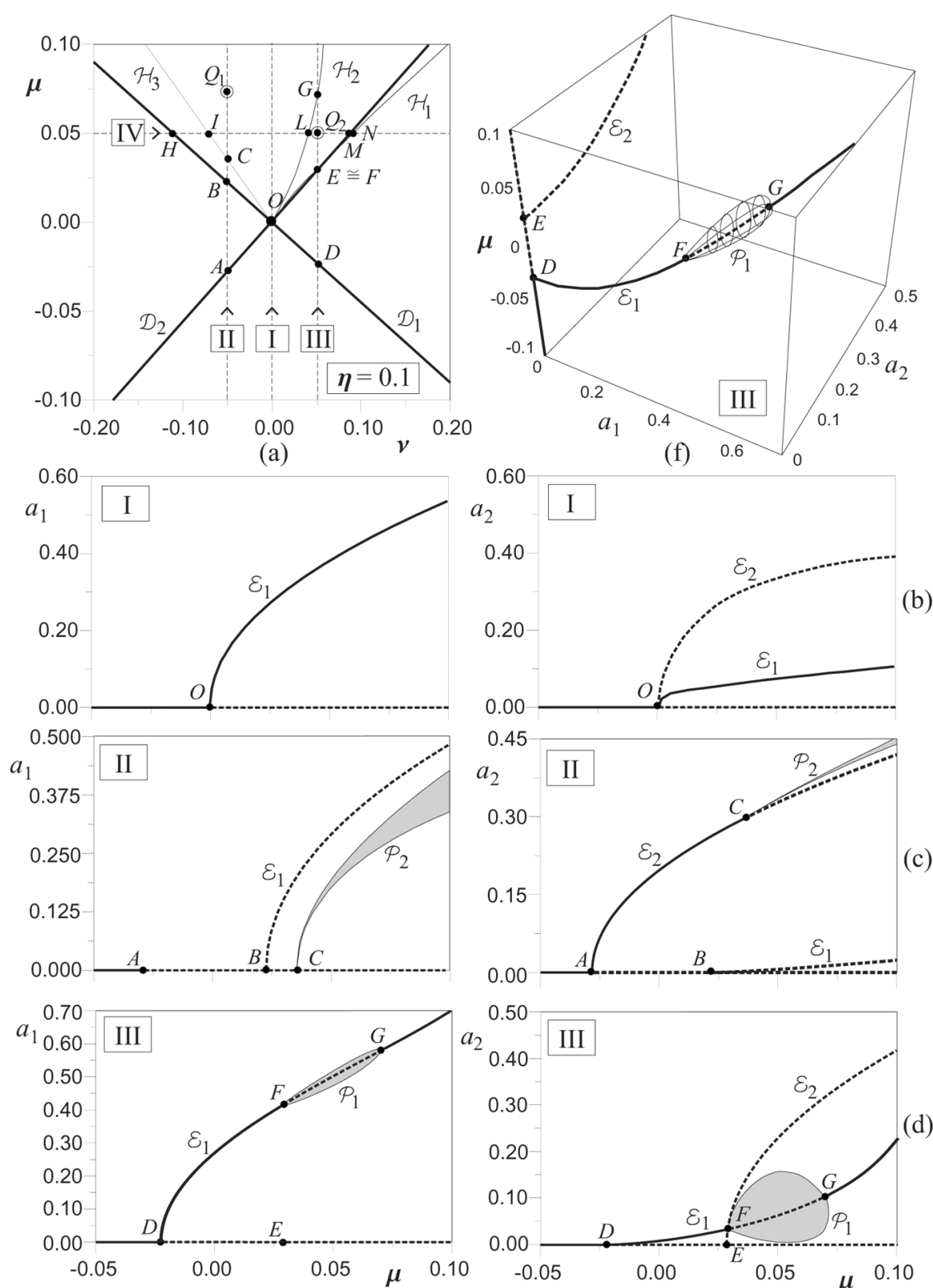

b)
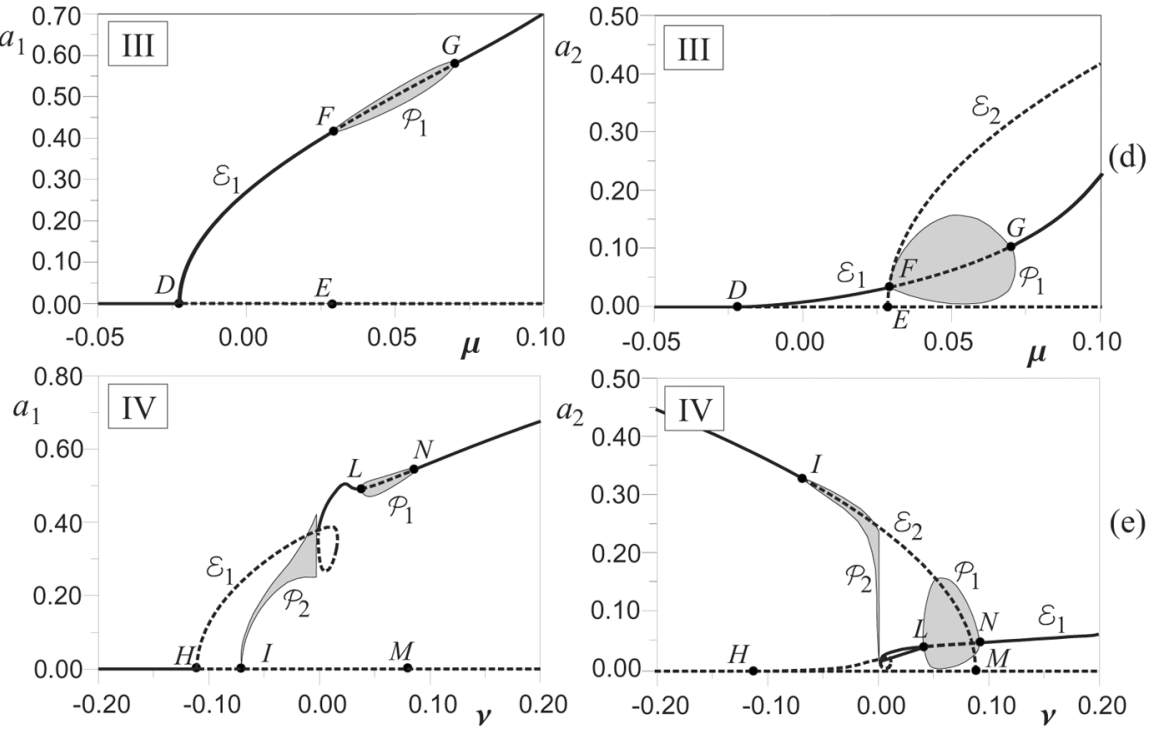

Figure 3. Bifurcation diagrams for the 1:3 resonance: (a) critical boundaries and selected paths; (b) to (e): bifurcation diagrams along paths I to IV; (f) three-dimensional bifurcation diagram for path III; $\mathcal{E}_{i}$ : equilibrium branches, $\mathcal{P}_{j}$ : periodic branches. 
To depict the scenario of the system's post-critical behavior around the bifurcation point $O$, a value of $\eta$ has been fixed in Figure $3_{a}$ and the problem analyzed in the plane of the two remaining parameters $\mu$ and $v$. There, the critical boundaries $\mathscr{D}_{1}$ and $\mathscr{D}_{2}$ at which the trivial solution loses stability through divergence are drawn, together with the three new boundaries $\mathscr{H}_{1}, \mathscr{H}_{2}$ (and $\mathscr{H}_{3}$ ) at which the bimodal (monomodal) solution undergoes a Hopf bifurcation. In Figure 3 a four straight paths are considered, along which the bifurcation diagrams of Figure $3 \mathrm{~b}$ to $3 \mathrm{e}$ are built up. Along pat I (Figure $3 \mathrm{~b}$ ), a double divergence takes place at $O$, from which two equilibrium paths, a stable path $\varepsilon_{1}$ and an unstable path $\varepsilon_{2}$, bifurcate. For negative $v$ (path II, Figure 3c), the two bifurcations split so that $\mathcal{E}_{2}$ first arises at $A$, and $\mathcal{E}_{1}$, then at $B$ (see also the points marked in Figure 3a). It should be noted that the stability is now exchanged with respect to path I and that $a_{2} \ll a_{1}$ in the bimodal $\mathscr{E}_{1}$ solution. At point $C, \mathcal{E}_{2}$ loses stability through a Hopf bifurcation and a stable family $\mathcal{P}_{2}$ of periodic motions, in which both the components $a_{1}$ and $a_{2}$ are involved, is triggered. The shaded areas in Figure $3 c$ denote the values spanned by the amplitudes during the periodic motion. For positive $v$ (path III, Figure $3 \mathrm{~d}$ ), $\mathcal{E}_{1}$ (bifurcating at $D$ ) precedes $\mathcal{E}_{2}$ (bifurcating at $E$ ); again, the first bifurcating branch is stable, the other unstable. At points $F$ and $G, \mathcal{E}_{1}$ first loses and then regains stability through two successive Hopf bifurcations, where a family of stable limit cycles $\mathcal{P}_{1}$ arises and disappears, respectively. A three-dimensional view of path III in the $\left(a_{1}, a_{2}, \mu\right)$-space is also given in Figure 3f. Along path IV (Figure 3a), in contrast to the former paths, the parameter $\mu$ is kept fixed and the parameter $v$ is varied. The relevant bifurcation diagram is displayed in Figure $3 \mathrm{e}$. The equilibrium paths $\varepsilon_{1}$ and $\varepsilon_{2}$ bifurcate from points $H$ and $M$, respectively, and are both unstable. However, $\mathcal{E}_{1}$ first regains stability at $v=0$ after a saddle-node bifurcation and then loses and again regains stability at $L$ and $N$, respectively. There, as in path III, stable limit cycles $\mathcal{P}_{1}$ appear and then disappear; therefore, curves $\mathscr{H}_{1}$ and $\mathscr{H}_{2}$ in Figure 3 a bound a region of existence of stable periodic solutions. An interesting phenomenon occurs at $I$ (Figure $3 \mathrm{e}$ ), where a family of limit cycles $\mathcal{P}_{2}$ bifurcates from $\mathcal{E}_{2}$, similarly to what happens at point $C$ along path II. However, unlike in the latter, when $v$ is increased and approaches zero, the limit cycle increases to include the saddle-node point (Figure 3e), while its period tends to infinity. A homoclinic bifurcation therefore takes places at $v=0$, since the limit cycle collides with the bimodal solution $\mathscr{E}_{1}$ at $v=0$. It turns out that the curves $\mathscr{H}_{3}$ and $v=0$ bound a region of limit cycles.

To describe fully the codimension-three bifurcation, the effect of the third control parameter $\eta$ must be investigated. The evolution of the Hopf boundaries $\mathscr{H}_{i}(i=1,2,3)$ for increasing $\eta$ 's is shown in Figure 4 . It is seen that $\mathscr{H}_{3}$ does not depend on $\eta$. In contrast $\mathscr{H}_{1}$ and $\mathscr{H}_{2}$ increasingly change their concavity with $\eta$, until they form a cusp that successively moves away from the origin.

To illustrate the shape of the limit cycles previously discussed, the projection on the $\left(a_{1}, a_{2}\right)$-plane of two trajectories approaching stable periodic solutions is shown in Figure 5. The values of the parameters correspond to the points $Q_{1}$ and $Q_{2}$ marked in Figure 3a, on path II and at the crossing of paths III and IV, respectively. According to Figure $3 \mathrm{c}$ the amplitude modulation at $Q_{1}$ is weak, while, according to Figures $3 \mathrm{~d}$ and $3_{e}$, the periodic motion at $Q_{2}$ is strongly modulated in the $a_{2}$-component.

A sketch of the phase-portraits around the bifurcations point $O$ is given in Figure 6 for a fixed $\eta$ and $\mu$ and varying $\nu$. The boundary curves $\mathscr{D}_{i}(i=1,2)$ and $\mathscr{H}_{j}(j=1,2,3)$ bound eight regions of the $(v, \mu)$-plane in which qualitatively different phase-portraits exist. These are represented in Figure 6 in the bidimensional space $\left(a_{1}, a_{2}\right)$, by ignoring the effects of the third state-variable (the phase-difference); in particular, crossings of the projected trajectories 

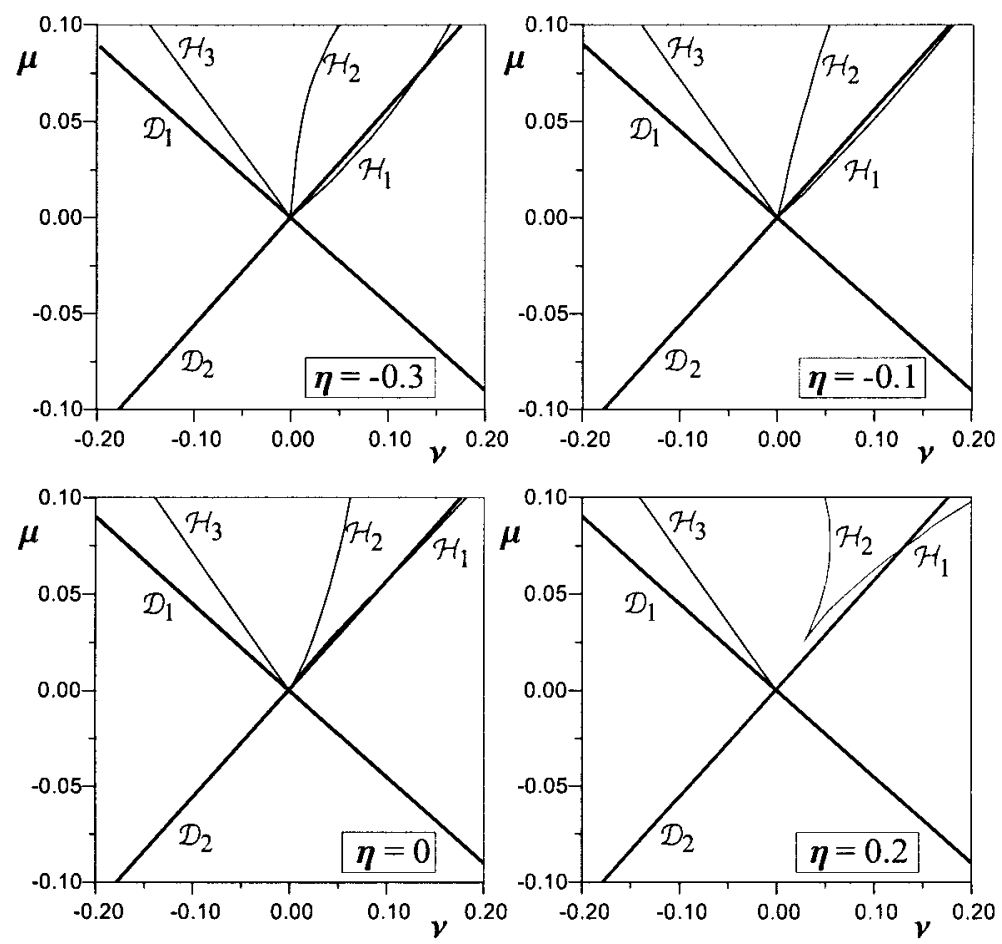

Figure 4. Evolution of the critical boundaries for $\eta$ varying for the 1:3 resonance.
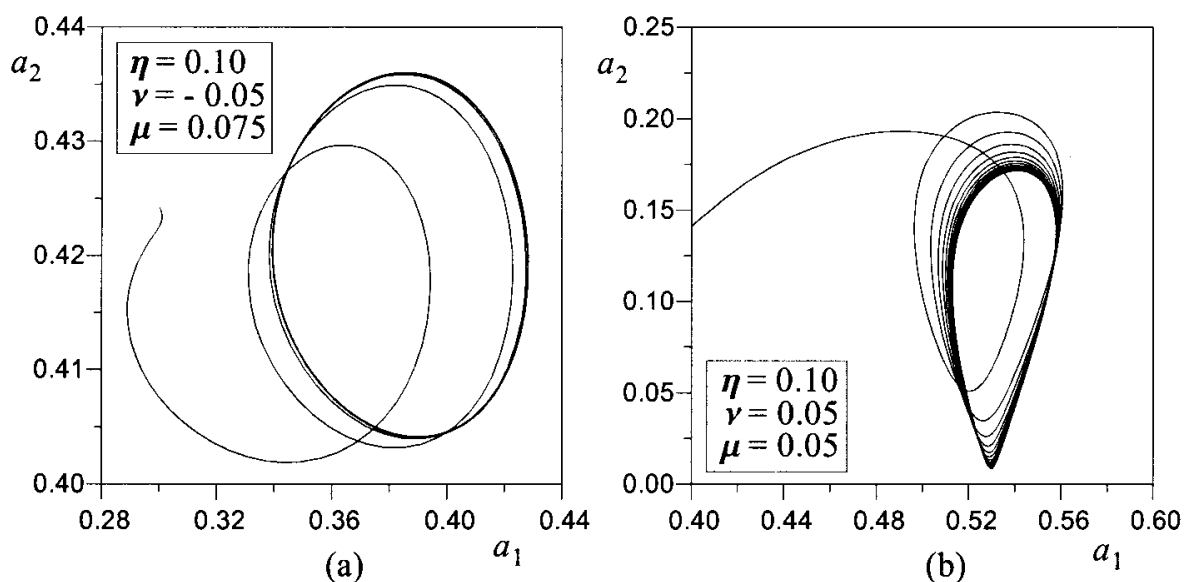

Figure 5. Trajectories approaching limit cycles for (a) the $Q_{1}$-point and (b) the $Q_{2}$-point marked in Figure 3 a.

are not shown and the flow is only sketched. By moving counterclockwise from region 1 to region 5, the following scenario is displayed: in region 1 the trivial solution $E_{0}$ is stable; in region 2, after a divergence bifurcation at $\mathcal{D}_{1}$, a stable equilibrium point $E_{1} \in \mathcal{E}_{1}$ arises; in region 3 , after the Hopf boundary $\mathscr{H}_{1}$ is crossed, $\mathscr{E}_{1}$ loses stability and a stable periodic solution $p_{1} \in \mathcal{P}_{1}$ is born; in region 4 , after a divergence at $\mathcal{D}_{2}$, an unstable equilibrium point $E_{2} \in \mathcal{E}_{2}$ arises on the $a_{2}$-axis; in region 5, after another Hopf bifurcation at $\mathscr{H}_{2}$, the limit limit cycle disappears and $\mathcal{E}_{1}$ regains stability. Moving clockwise from region 1 to region 8 : 


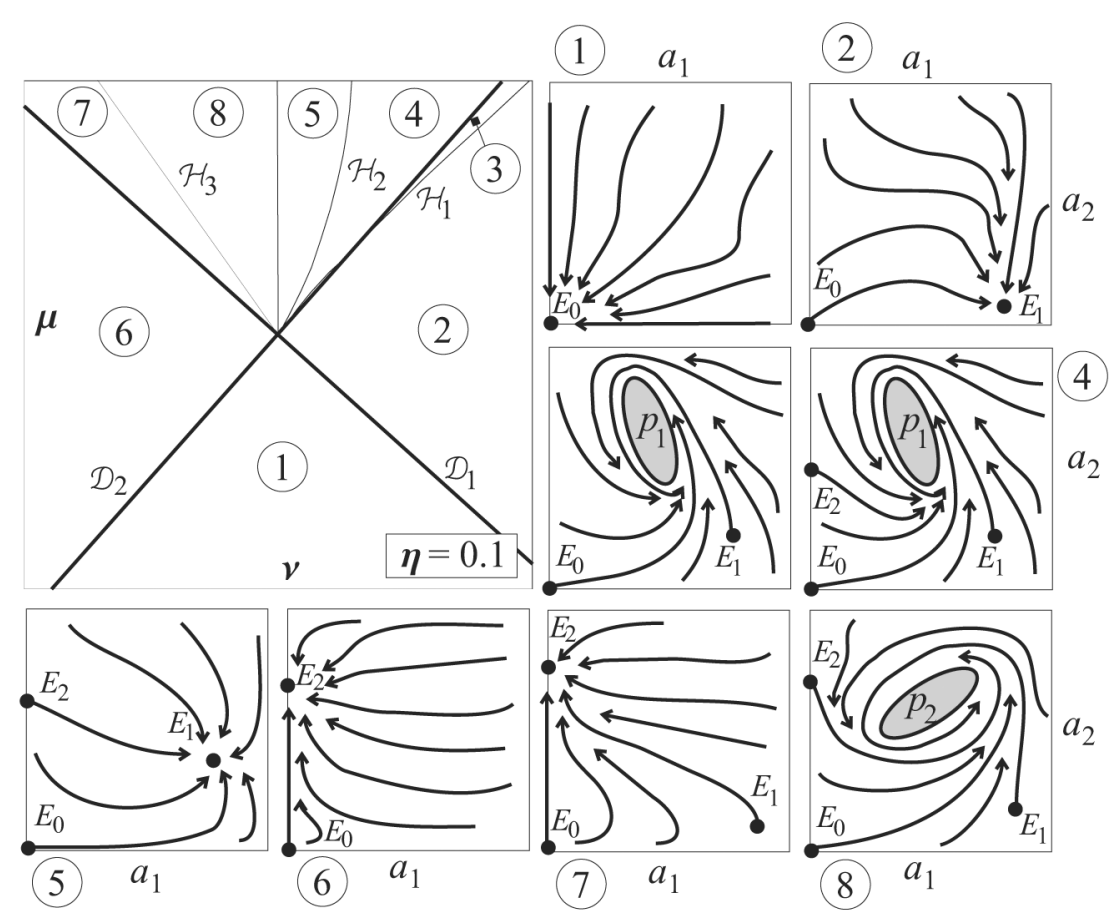

Figure 6. Phase-portraits scenario for the 1:3 resonance.
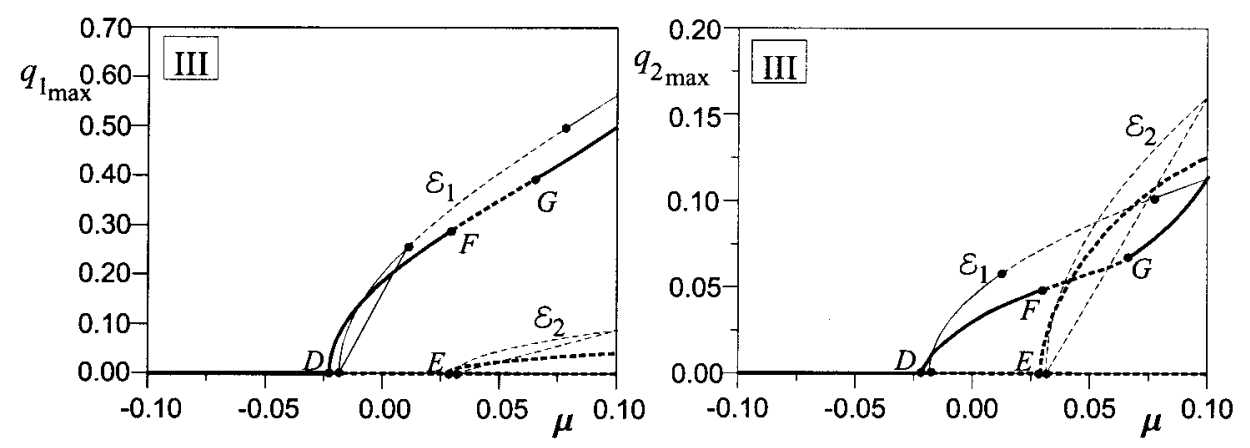

Figure 7. Comparison between asymptotic (heavy lines) and exact (thin lines) periodic $\left(q_{1}, q_{2}\right)$-solutions along path III of Figure 3d.

in region 6 , after crossing the divergence boundary $\mathscr{D}_{2}$, the stable equilibrium point $E_{2} \in \varepsilon_{2}$ is born; in region 7 , due to a second divergence occurring at $\mathscr{D}_{1}$, an unstable point $E_{1} \in \mathcal{E}_{1}$ appears; in region 8 , after the Hopf bifurcation occurs at $\mathscr{H}_{3}$, a stable periodic solution $p_{2} \in \mathcal{P}_{2}$ arises, meaning that $E_{2}$ loses stability. The transition of the phase-portraits from region 8 to region 5 is a consequence of a homoclinic bifurcation occurring on the positive $\mu$-axis, at which the periodic solution disappears while, simultaneously, a saddle-node bifurcation modifies the stability of the equilibrium at $E_{1}$. It should be noted that in each region only one attractor exists, namely an equilibrium point (in regions $1,2,5,6,7$ ) or a limit cycle (in regions $3,4,8)$.

Finally, in order to validate the perturbation approach, the results furnished by the solution of the bifurcation equations are compared in Figure 7 with direct numerical integrations of 

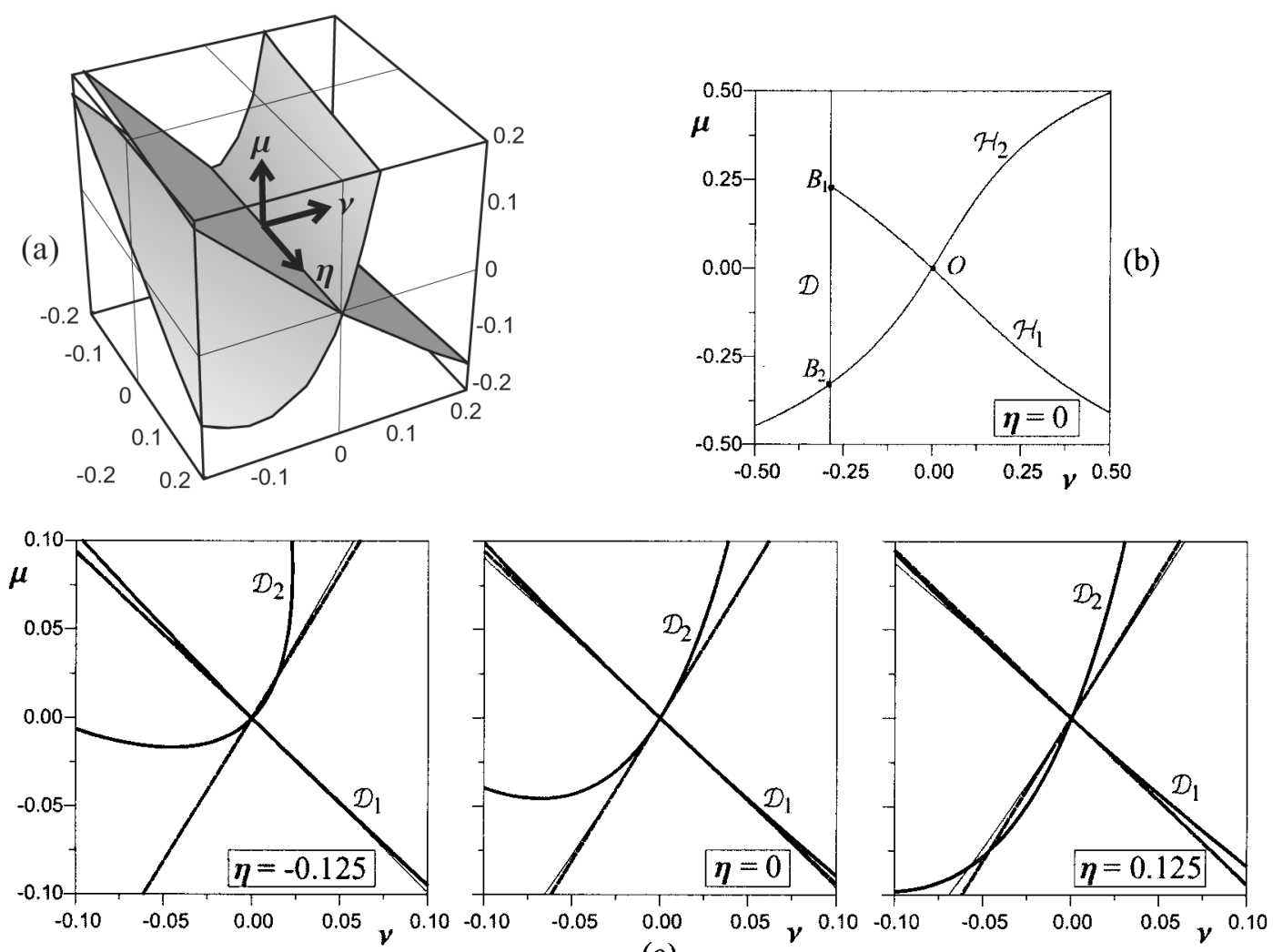

(c)

Figure 8. Linear stability diagrams for the 1:2 resonance: (a) three-dimensional view of the asymptotic boundaries; (b) exact boundaries; (c) comparison between second-order (continuos heavy lines), first-order (dashed heavy lines) asymptotic solutions and exact (thin lines) solutions.

the equations of motions. Path III of Figure $3 \mathrm{~d}$ is again considered and use is made of Equations (11) and (21) to express $\mathbf{x}$ at the $\varepsilon^{2}$-order. Then the maximum values of the Lagrangian coordinates $q_{1}$ and $q_{2}$ (heavy lines in Figure 7) are evaluated and compared with numerical results (thin lines). Only steady-state amplitude solutions (i.e. periodic state variables solutions) are compared. It should be noted that, due to the transformations from the modal amplitude to the Lagrangian coordinates, the monomodal equilibrium $\varepsilon_{2}$ has a nonzero component in both $q_{1}$ and $q_{2}$. It can be seen that, although significant quantitative differences are present, the first-order perturbation solution captures all the qualitative aspects of the nonlinear behavior.

\subsection{1:2 RESONANCE}

The foregoing analysis is repeated for the 1:2 resonance. In Figure 8 the linear stability diagrams obtained by solving both the linearized bifurcation equations (Figure 8a) and the equations of motion (Figure 8b) are shown and then compared in Figure 8c. Differently from the 1:3 resonance (Figure 2a and Equation (22)), the asymptotic critical boundaries (Figure 8a) have strong curvatures, due to the nonlinear dependence of Equation (17) on the control parameters, as caused by the reconstitution procedure; moreover, the parameter $\eta$ strongly affects the critical boundaries. The exact curves of Figure $8 \mathrm{~b}$ confirm this curvature at the bifurcation 
point $O$. However, when the asymptotic (continuous heavy lines) and the exact (thin lines) curves are compared (Figure 8c), while the $\mathscr{D}_{1}$-boundary is accurately described from the approximated solutions, a large error is found for $\mathscr{D}_{2}$, although the exact curvature at $O$ is captured. In contrast, if only the linear terms in $\mu$ are taken in the bifurcation equation (i.e., if the perturbation expansion is truncated to the $\varepsilon^{2}$-order), the dashed curves shown in Figure $8 \mathrm{c}$ are found closer to the exact lines. However, as confirmed in the forthcoming analysis, the $\varepsilon^{3}$-order terms play an important role in describing the postcritical behavior and cannot be neglected so that, for the sake of consistency, the complete bifurcation Equations (17) are used in the analysis. The possibility of neglecting the $\boldsymbol{\mu}^{2} \mathbf{A}$-terms while keeping the equal order $\mathbf{A}^{3}$-terms, is not explored here; it will be the subject of a future study.

A number of bifurcation diagrams for different values of the parameters are shown in Figure 9. Several divergence boundaries $\mathscr{D}_{i}$ and Hopf boundaries $\mathscr{H}_{j}(i, j=1,2,3)$ on the $(\nu, \mu)$-plane exist for a fixed $\eta$ (Figure 9a). At $\mathscr{D}_{1}$ and $\mathscr{D}_{2}$, a branch $\mathscr{E}_{1}:=\left(a_{1}, a_{2}\right)$ (periodic solution $\mathbf{x}$ of frequency close to $\left.\omega_{10}\right)$ and a branch $\varepsilon_{2}:=\left(0, a_{2}\right)$ (periodic solution $\mathbf{x}$ of frequency close to $\omega_{20}$ ) respectively, arise (Figure 9e). Solution $\varepsilon_{1}$ undergoes two Hopf bifurcations at $\mathscr{H}_{1}$ and $\mathscr{H}_{2}$; solution $\mathcal{E}_{2}$ undergoes a divergence bifurcations at $\mathcal{D}_{3}$, where a new branch of equilibrium points $\mathcal{E}_{3}:=\left(a_{1}, a_{2}\right)$ is born; this latter exhibits a Hopf bifurcation at $\mathscr{H}_{3}$. Along path I (Figure 9b) a double divergence occurs at $O$, with $\varepsilon_{1}$ being stable and $\varepsilon_{2}$ unstable. Along path II (Figure 9c) the monomodal $\mathscr{E}_{2}$ first occurs at $A$, being initially stable; it then loses stability at $B$ by divergence, where a stable branch $\varepsilon_{3}$ of bimodal solutions is borne. When $C$ is reached, the unstable $\varepsilon_{1}$ also bifurcates from the trivial solution. Along path III (Figure $9_{d, e}$ ) the richest bifurcation scenario is attained. At $D$ the stable branch $\varepsilon_{1}$ bifurcates from the trivial solution. It first loses and then regains stability at $E$ and $I$ respectively, where Hopf bifurcations trigger two families of stable $\mathcal{P}_{1 l}$ and unstable $\mathcal{P}_{1 u}$ limit cycles. At $F$ the unstable $\mathcal{E}_{2}$-branch also bifurcates from the trivial solution; it undergoes a divergence at $H$ that gives rise to the unstable $\mathcal{E}_{3}$-branch; after a saddle-node, $\mathcal{E}_{3}$ regains stability, but again loses it at $G$, where a family $\mathcal{P}_{3}$ of stable limit cycles arises. The three families of limit cycles borned at $E, G$, and $I$ suddenly disappear for increasing $\mu$ because of the occurrence of homoclinic bifurcations, signaled by the tendency to infinity of their periods. In particular $\mathcal{P}_{1 l}$ collide with $\mathscr{E}_{1}$, while $\mathcal{P}_{3}$ and $\mathcal{P}_{1 u}$ collide with $\mathcal{E}_{2}$.

To analyze the effect of $\eta$ on the postcritical behavior, a new value is fixed in Figure 10. Only the boundaries $\mathscr{H}_{1}$ and $\mathscr{H}_{2}$ are found, while $\mathscr{D}_{3}$ and $\mathscr{H}_{3}$ disappear (Figure 10a). Path III of Figure 9d changes as shown in Figure 10b, i.e. it is similar to that existing in the 1:3 resonance (Figure $3 \mathrm{~d}$ ). The lack of $\mathscr{D}_{3}$ prevents the birth of $\mathcal{E}_{3}$ bifurcating from $\mathcal{E}_{1}$ and, consequently, that of the limit cycles at $\mathscr{H}_{3}$. The limit cycles triggered at $\mathscr{H}_{1}$ therefore die at $\mathscr{H}_{2}$.

The evolution with $\eta$ of the critical boundaries $\mathscr{D}_{i}$ and $\mathscr{H}_{j}(i, j=1,2,3)$ is analyzed in Figure 11. It is seen that, starting from the maximum values of $\eta$, the $v>0$ branch of $\mathscr{D}_{3}$ progressively changes concavity and then collapses on $\mathscr{D}_{2}$. Simultaneously, the boundary $\mathscr{H}_{2}$ approaches $\mathscr{H}_{3}$ until it collapses on it. Thus, $\mathscr{H}_{3}$ disappears together with $\mathscr{D}_{3}$, since it is the locus of Hopf bifurcations occurring along the branches generated by $\mathscr{D}_{3}$. Once $\mathscr{D}_{3}$ and $\mathscr{H}_{3}$ have disappeared, $\mathscr{H}_{1}$ and $\mathscr{H}_{2}$ detach from the bifurcation point.

As an example, the projection on the $\left(a_{1}, a_{2}\right)$-plane of the trajectories approaching two stable limit cycles at points $Q_{1}$ and $Q_{2}$ of Figure 9a and 10a are shown in Figure $12_{a, b}$ respectively. It highlights the strong modulation of both the amplitudes at $Q_{1}$ and of $a_{2}$ at $Q_{2}$. 

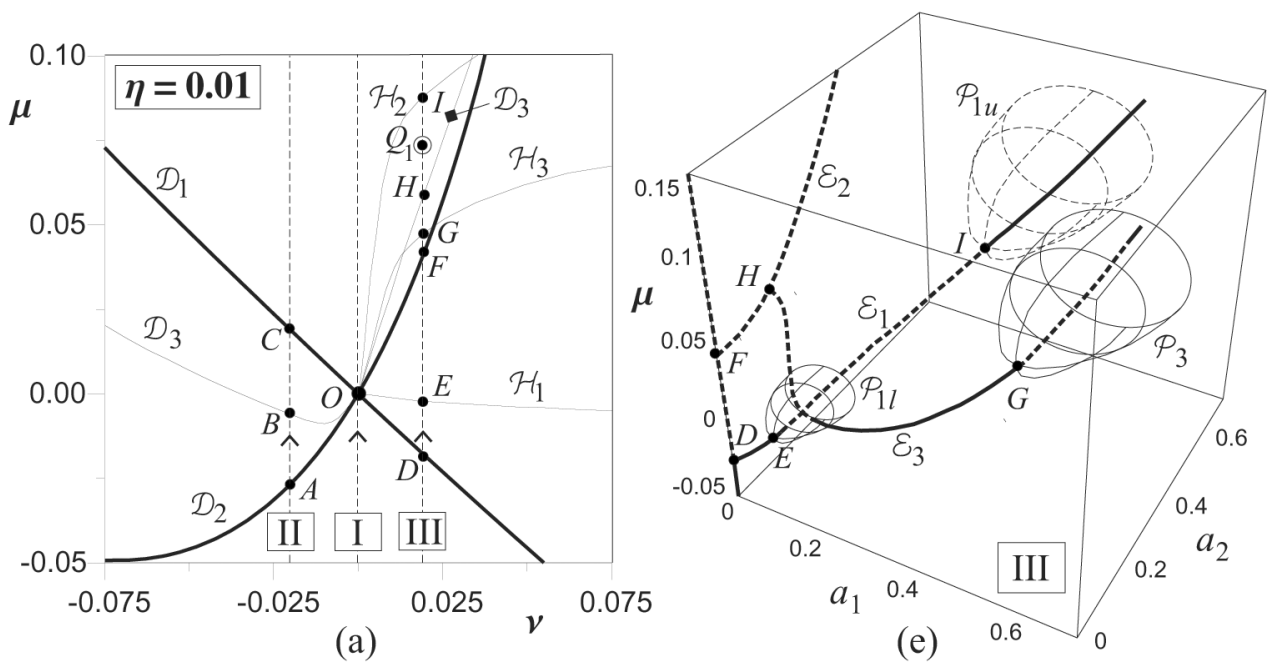

(a)

(e)
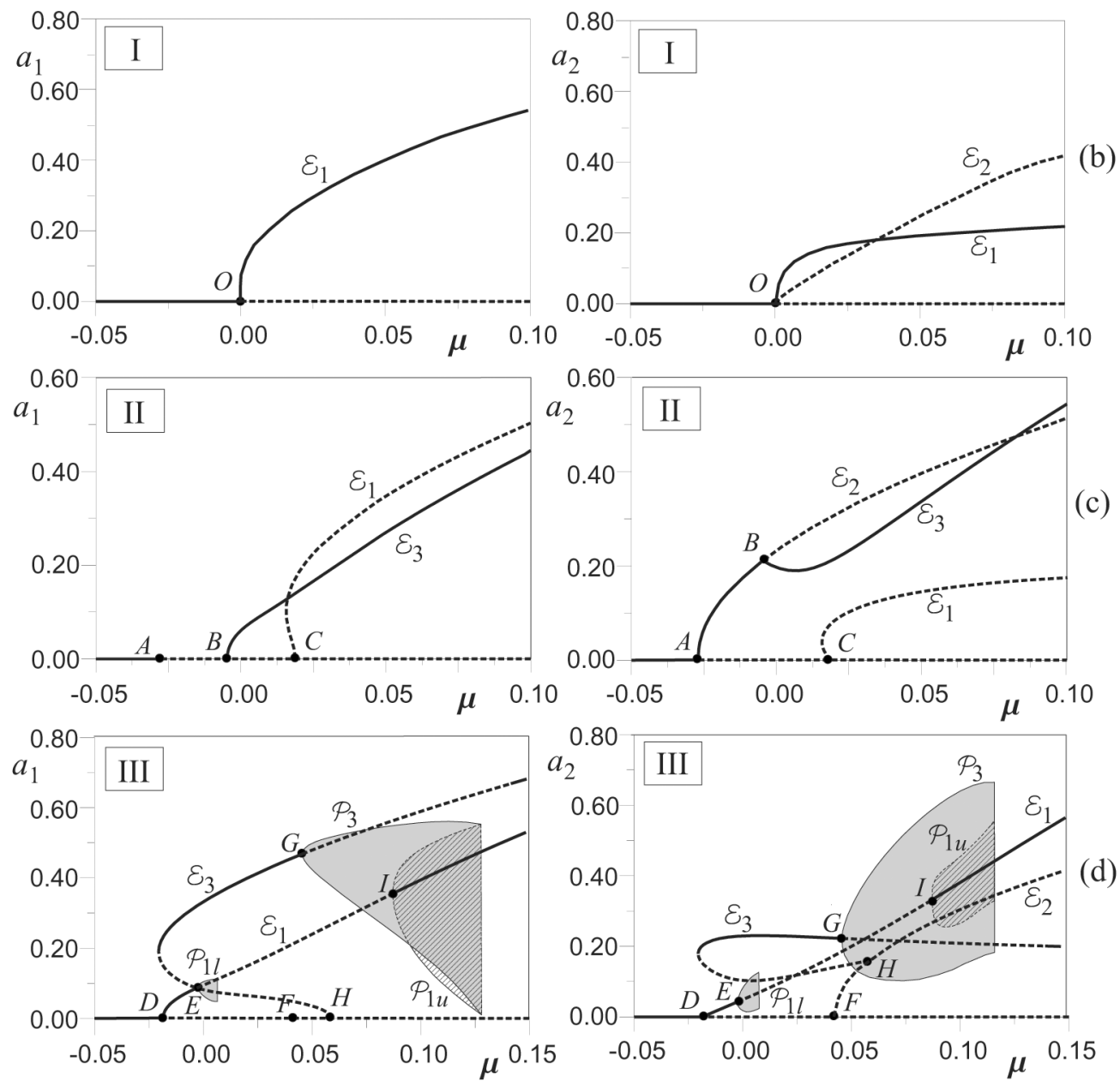

Figure 9. Bifurcation diagrams for the 1:2 resonance: (a) critical boundaries and selected paths; (b) to (d): bifurcation diagrams along paths I to III; (f) three-dimensional bifurcation diagram for path III; $\varepsilon_{i}$ : equilibrium branches, $\mathcal{P}_{j}$ : periodic branches. 


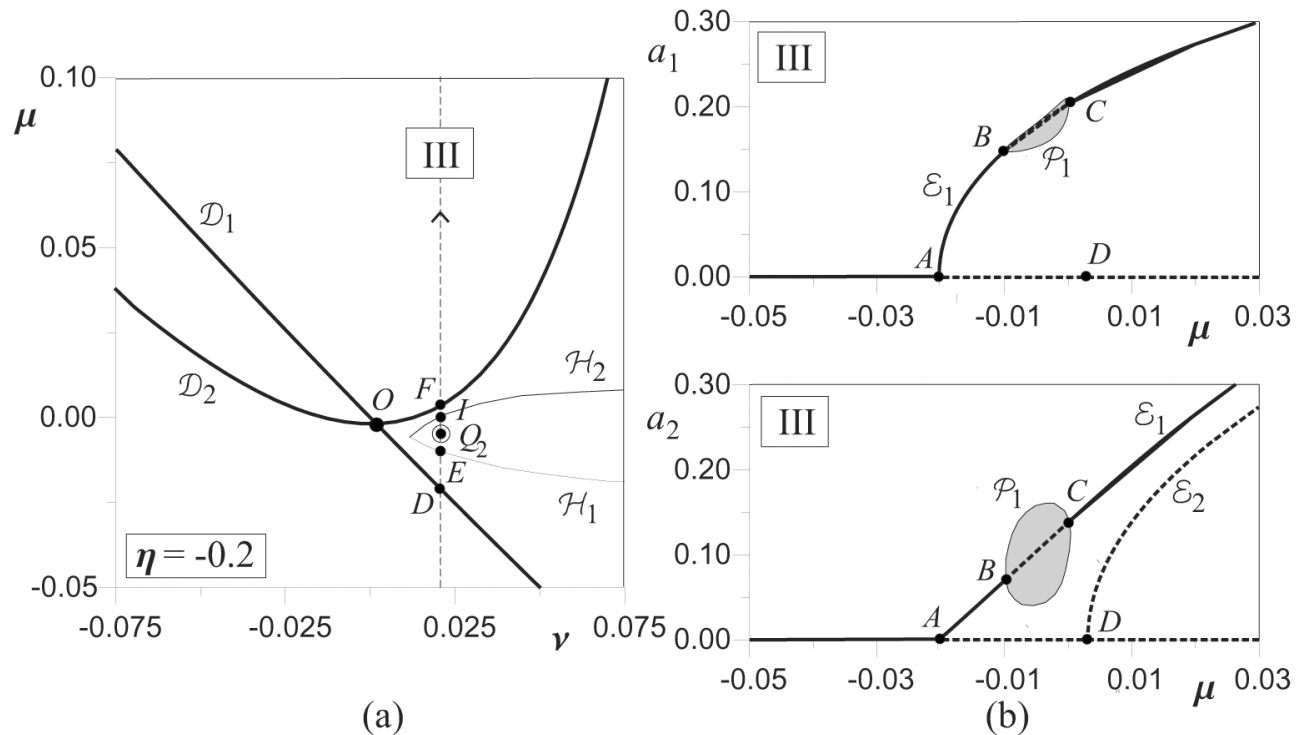

Figure 10. Bifurcation diagrams for path III of Figure 3d when $\eta$ is modified: (a) boundary curves; (b) bifurcation diagram; $\mathcal{E}_{i}$ : equilibrium branches, $\mathcal{P}_{j}$ : periodic branches.
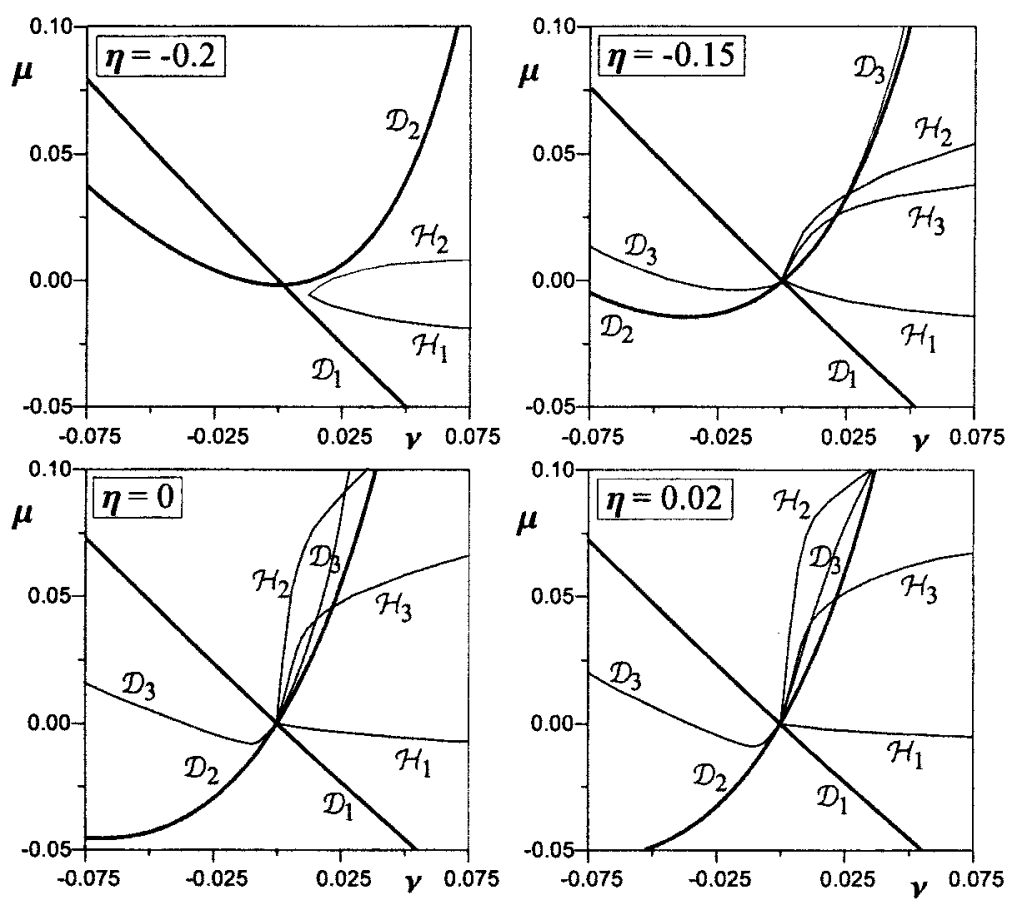

Figure 11. Evolution of the critical boundaries for $\eta$ varying; 1:2 resonance. 


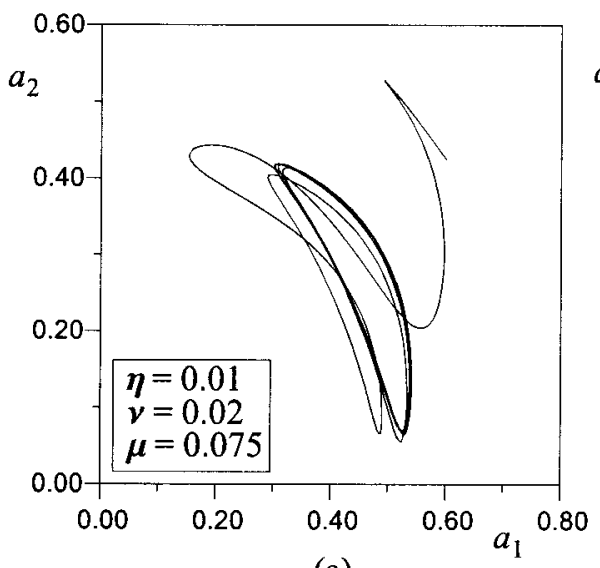

(a)

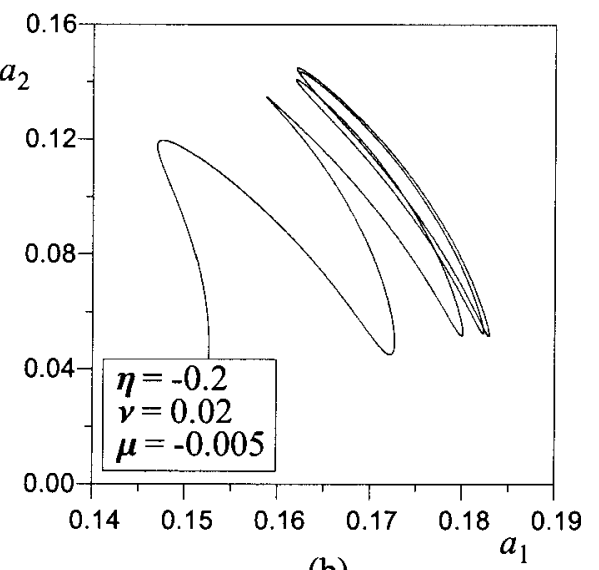

(b)

Figure 12. Trajectories approaching limit cycles for (a) the $Q_{1}$-point and (b) the $Q_{2}$-point marked in Figures 9 a and $10 \mathrm{a}$, respectively.

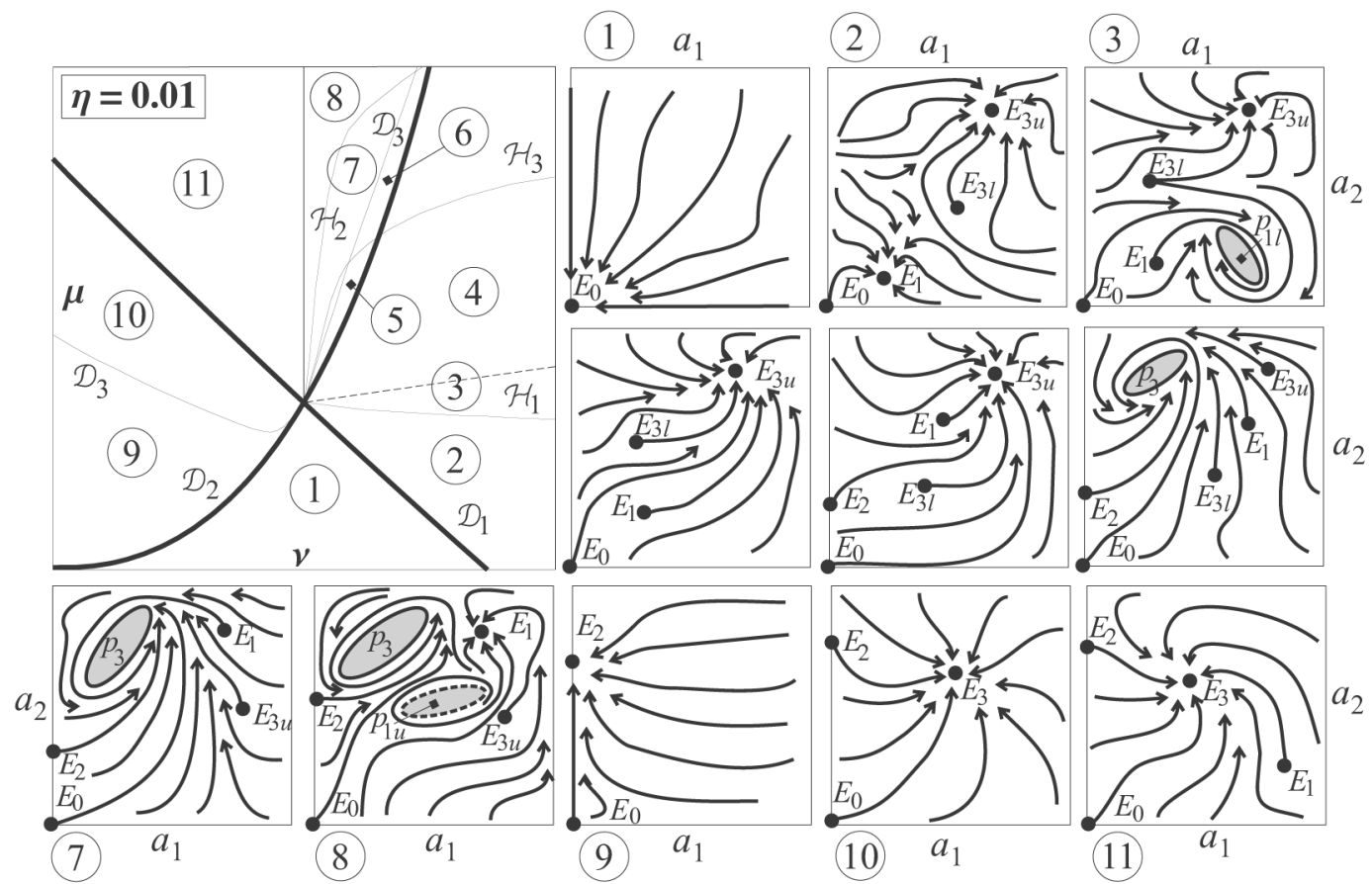

Figure 13. Phase-portraits scenario for the 1:2 resonance.

The typical phase-portraits relevant to several regions of the $(v, \mu)$-plane are sketched in Figure 13, where the same value of $\eta$ in Figure 9 is taken. By moving counterclockwise from region 1 to region 8, the following evolution is observed (see also path III in Figures $9_{d, e}$ ): in region I the trivial solution is stable; in region 2, there exists a stable equilibrium point $E_{1} \in$ $\mathcal{E}_{1}$, originated by the divergence at $\mathscr{D}_{1}$, and a couple of unstable/stable points $\left(E_{3 l}, E_{3 u}\right) \in \mathcal{E}_{3}$, originated at $\mathscr{D}_{3}$; in region 3 , after a Hopf bifurcation at $\mathscr{H}_{1}$, a stable limit cycle $p_{1 l} \in \mathcal{P}_{1 l}$ arises and then disappears in region 4 , because of a homoclinic bifurcation; in region 5 , due to the divergence at $\mathscr{D}_{2}$, an unstable equilibrium point $E_{2} \in \mathcal{E}_{2}$ appears; by crossing $\mathscr{H}_{3}$, a stable 

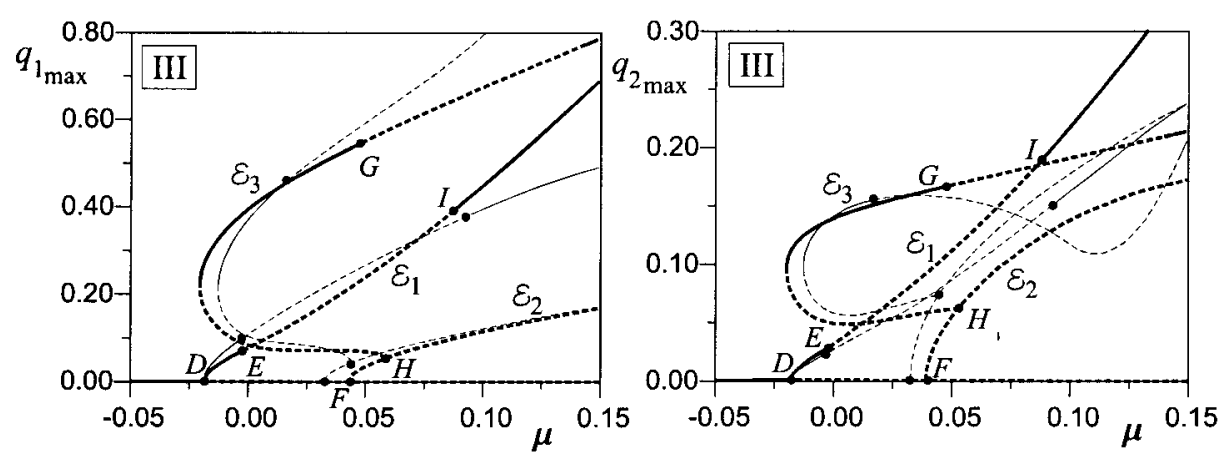

Figure 14. Comparison between asymptotic (heavy lines) and exact (thin lines) periodic $\left(q_{1}, q_{2}\right)$-solutions along path III of Figure 9d.

limit cycle $p_{3} \in \mathcal{P}_{3}$ is generated in region 6 ; in region 7 the $E_{3 l}$-point disappears, once $\mathscr{D}_{3}$ is crossed; in region 8 an unstable limit cycle $p_{1 u} \in \mathcal{P}_{1 u}$ arises, triggered by the Hopf bifurcation at $\mathscr{H}_{2}$. Moving clockwise from region 1 to region 11, the following evolution is observed: in region 9 , caused by the divergence at $\mathscr{D}_{2}$, the stable equilibrium point $E_{2} \in \mathcal{E}_{2}$ appears; in region 10 , after $\mathscr{D}_{3}$ is crossed, $E_{2}$ loses stability and a new stable point $E_{3} \in \varepsilon_{3}$ is born; finally, in region 11 , the further unstable point $E_{1} \in \mathcal{E}_{1}$ appears caused by the divergence at $\mathscr{D}_{1}$. The transition from region 11 to region 8 occurs through the homoclinic boundary line $v=0$, $\mu>0$, which causes the two cycles to disappear; moreover, the stable equilibrium point $E_{1}$ loses stability because of a saddle-node bifurcation. In conclusion, the 1:2 case presents a richer scenario than the 1:3 case, since regions are found where only one attractor exists (an equilibrium point in regions $1,5,9,10,11$ or a cycle in regions 6,7$)$ or two attractors are in competition (two equilibrium points in regions 2,4 and an equilibrium point and a cycle in regions 3 and 8 ).

Perturbative and exact solutions are finally compared in Figure 14 in terms of maximum values assumed by the Lagrangian coordinates along path III of Figure 9. Comparison is limited to periodic solutions. Only quantitative differences appear, above all along the unstable branch subsequent to point $G$. The accordance is however satisfactory.

\section{Conclusions}

The bifurcation equations of a general $n$-dimensional system undergoing 1:2 or 1:3 resonant double Hopf bifurcations are obtained in closed form in terms of the derivatives of the original vector field evaluated at the bifurcation point. By truncating the analysis at the $\varepsilon^{3}$-order, where $\varepsilon$ is a perturbation parameter of the order of magnitude of the amplitude of motion, first-order bifurcation equations are obtained in the 1:3 case, and second-order bifurcation equations in the 1:2 case. In the latter case, use is made of the reconstitution procedure [2]. A mixed polar-Cartesian representation for the amplitudes is used [14], in order to obtain a set of three bifurcation equations in standard normal form, in contrast with the usual pure polar representation leading to nonstandard form equations. The equations thus obtained, unlike the latter, are well-suited for analysis by software packages such as AUTO [15], which is used here.

As an example, a two-degrees-of-freedom mechanical system is studied, loaded by wind forces which trigger self-excited vibrations. For a suitable choice of the three control parame- 
ters (two system parameters and the wind velocity, considered as a distinguished parameter) both 1:2 and 1:3 resonant Hopf bifurcations can occur. By varying the set of control parameters, the neighborhood of the bifurcation point is investigated. The linear stability surfaces evaluated by the asymptotic procedure are found to be in excellent accordance with the exact surfaces in the 1:3 case, while some quantitative descrepancies are noticed in the 1:2 case, due to the strong curvature of the surfaces at the bifurcation point. Bifurcation diagrams are built up to give a full description of the codimension-3 bifurcation: they reveal the existence of periodic and periodically modulated solutions, as well as homoclinic bifurcations. Phaseportraits on regions of a two-dimensional parameter space are sketched. It is found that while in the 1:3 case only one attractor exists, namely, a periodic or a periodically modulated motion, in the 1:2 case, two attractors may compete each other. The comparison between asymptotic and numerically exact results reveals a qualitatively excellent agreement and a satisfactory quantitative accordance.

\section{Appendix A}

The row vectors and coefficients $\mathbf{C}_{j \mu}(j=1,2)$ and $C_{j h}$ appearing in Equation (13) are defined as follows

$$
\mathbf{C}_{j \mu} \widehat{\boldsymbol{\mu}}=\mathbf{v}_{j}^{H} \mathbf{F}_{\mathbf{x} \mu}^{0} \mathbf{u}_{j} \widehat{\boldsymbol{\mu}}, \quad C_{\overline{1} 2}=\mathbf{v}_{1}^{H} \mathbf{F}_{\mathbf{x x}}^{0} \overline{\mathbf{u}}_{1} \mathbf{u}_{2}, \quad C_{11}=\mathbf{v}_{2}^{H} \mathbf{F}_{\mathbf{x x}}^{0} \mathbf{u}_{1}^{2} .
$$

The vectors $\mathbf{z}_{r s}$ 's and $\mathbf{z}_{r \bar{s}}$ ' $(r, s=1,2) \in \mathbb{C}^{n}$ appearing in Equations (14) are obtained by solving linear nonsingular algebraic equations

$$
\begin{aligned}
& \left(i p \omega_{1} \mathbf{E}+i q \omega_{2} \mathbf{E}-\mathbf{F}_{\mathbf{x}}^{0}\right) \mathbf{z}_{r s}=\mathbf{F}_{\mathbf{x x}}^{0} \mathbf{u}_{r} \mathbf{u}_{s}, \\
& \left(i p \omega_{1} \mathbf{E}+i q \omega_{2} \mathbf{E}-\mathbf{F}_{\mathbf{x}}^{0}\right) \mathbf{z}_{r \bar{s}}=\mathbf{F}_{\mathbf{x x}}^{0} \mathbf{u}_{r} \overline{\mathbf{u}}_{s},
\end{aligned}
$$

in which $p$ and $q$ are the real coefficients of $\omega_{1}$ and $\omega_{2}$, respectively, appearing in the associated exponential functions of Equation (14), while the vectors and matrices $\mathbf{Z}_{j h}$ and $\mathbf{Z}_{j \mu}$ are obtained by solving the following singular linear algebraic equations

$$
\begin{aligned}
& \left(i \omega_{1} \mathbf{E}-\mathbf{F}_{\mathbf{x}}^{0}\right) \mathbf{Z}_{\overline{1} 2}=2\left[\mathbf{F}_{\mathbf{x x}}^{0} \overline{\mathbf{u}}_{1} \mathbf{u}_{2}-\mathbf{v}_{1}^{H}\left(\mathbf{F}_{\mathbf{x} x}^{0} \overline{\mathbf{u}}_{1} \mathbf{u}_{2}\right) \mathbf{u}_{1}\right], \\
& \left(i \omega_{2} \mathbf{E}-\mathbf{F}_{\mathbf{x}}^{0}\right) \mathbf{Z}_{11}=\left[\mathbf{F}_{\mathbf{x x}}^{0} \mathbf{u}_{1}^{2}-\mathbf{v}_{2}^{H}\left(\mathbf{F}_{\mathbf{x x}}^{0} \mathbf{u}_{1}^{2}\right) \mathbf{u}_{2}\right], \\
& \left(i \omega_{j} \mathbf{E}-\mathbf{F}_{\mathbf{x}}^{0}\right) \mathbf{Z}_{j \mu} \widehat{\boldsymbol{\mu}}=2\left[\mathbf{F}_{\mathbf{x} \mu}^{0} \mathbf{u}_{j} \widehat{\boldsymbol{\mu}}-\mathbf{v}_{j}^{H}\left(\mathbf{F}_{\mathbf{x} \mu}^{0} \mathbf{u}_{j} \widehat{\boldsymbol{\mu}}\right) \mathbf{u}_{j}\right] .
\end{aligned}
$$

Since $\left(i \omega_{j} \mathbf{E}-\mathbf{F}_{\mathbf{x}}^{0}\right)$ is singular, the solutions of Equations (28) are not unique. To avoid indeterminacies, an additional condition must be enforced, e.g. by imposing $\mathbf{v}_{j}^{H} \mathbf{Z}=0$.

The coefficients $C_{i j h}$, the row vectors $\mathbf{C}_{i j \mu}$ and the matrices $\mathbf{C}_{i \mu \mu}$ appearing in Equation (15) are given by

$$
\begin{aligned}
C_{12 \overline{2}}= & \mathbf{v}_{1}^{H}\left(-C_{\overline{1} 2} \mathbf{Z}_{\overline{1} 2}+\mathbf{F}_{\mathbf{x x}}^{0} \mathbf{z}_{2 \overline{2}} \mathbf{u}_{1}+\mathbf{F}_{\mathbf{x x}}^{0} \overline{\mathbf{z}}_{2 \overline{2}} \mathbf{u}_{1}+\mathbf{F}_{\mathbf{x x}}^{0} \mathbf{z}_{\overline{1} 2} \mathbf{u}_{2}+\mathbf{F}_{\mathbf{x x}}^{0} \mathbf{z}_{12} \overline{\mathbf{u}}_{2}\right. \\
& +2 \mathbf{F}_{\mathbf{x x x}}^{0} \mathbf{u}_{1} \mathbf{u}_{2} \overline{\mathbf{u}}_{2} \\
C_{11 \overline{1}}= & \mathbf{v}_{1}^{H}\left(-C_{11} \mathbf{Z}_{\overline{1} 2}+\mathbf{F}_{\mathbf{x x}}^{0} \mathbf{z}_{1 \overline{1}} \mathbf{u}_{1}+\mathbf{F}_{\mathbf{x x}}^{0} \overline{\mathbf{z}}_{1 \overline{1}} \mathbf{u}_{1}+\mathbf{F}_{\mathbf{x x}}^{0} \mathbf{z}_{11} \overline{\mathbf{u}}_{1}+\mathbf{F}_{\mathbf{x x x}}^{0} \mathbf{u}_{1}^{2} \overline{\mathbf{u}}_{1}\right) \\
C_{1 \overline{1} 2}= & \mathbf{v}_{2}^{H}\left(-2 C_{\overline{1} 2} \mathbf{Z}_{11}+\mathbf{F}_{\mathbf{x x}}^{0} \mathbf{Z}_{\overline{1} 2} \mathbf{u}_{1}+\mathbf{F}_{\mathbf{x x}}^{0} \mathbf{z}_{12} \overline{\mathbf{u}}_{1}+\mathbf{F}_{\mathbf{x x}}^{0} \mathbf{z}_{1 \overline{1}} \mathbf{u}_{2}\right. \\
& \left.+\mathbf{F}_{\mathbf{x x}}^{0} \overline{\mathbf{z}}_{1 \overline{1}} \mathbf{u}_{2}+2 \mathbf{F}_{\mathbf{x x x}}^{0} \mathbf{u}_{1} \overline{\mathbf{u}}_{1} \mathbf{u}_{2}\right) \\
C_{22 \overline{2}}= & \mathbf{v}_{2}^{H}\left(\mathbf{F}_{\mathbf{x x}}^{0} \mathbf{z}_{2 \overline{2}} \mathbf{u}_{2}+\mathbf{F}_{\mathbf{x x}}^{0} \overline{\mathbf{z}}_{2 \overline{2}} \mathbf{u}_{2}+\mathbf{F}_{\mathbf{x x}}^{0} \mathbf{z}_{22} \overline{\mathbf{u}}_{2}+\mathbf{F}_{\mathbf{x x x}}^{0} \mathbf{u}_{2}^{2} \overline{\mathbf{u}}_{2}\right)
\end{aligned}
$$




$$
\begin{aligned}
\mathbf{C}_{\overline{1} 2 \mu} \widehat{\boldsymbol{\mu}}= & { }_{1 \mu} \mathbf{Z}_{\overline{1} 2}-\mathbf{C}_{2 \mu} \mathbf{Z}_{\overline{1} 2}+\mathbf{F}_{\mathbf{x x}}^{0} \mathbf{Z}_{2 \mu} \overline{\mathbf{u}}_{1}+\mathbf{F}_{\mathbf{x x}}^{0} \overline{\mathbf{Z}}_{1 \mu} \mathbf{u}_{2} \\
& \left.+\mathbf{F}_{\mathbf{x} \mu}^{0} \mathbf{Z}_{\overline{\mathbf{1}} 2}+2 \mathbf{F}_{\mathbf{x x} \mu}^{0} \overline{\mathbf{u}}\right)_{1} \mathbf{u}_{2} \widehat{\boldsymbol{\mu}} \\
\mathbf{C}_{11 \mu} \widehat{\boldsymbol{\mu}}= & \mathbf{v}_{2}^{H}\left(C_{11} \mathbf{Z}_{2 \mu}-2 \mathbf{C}_{1 \mu} \mathbf{Z}_{11}+\mathbf{F}_{\mathbf{x x}}^{0} \mathbf{Z}_{1 \mu} \mathbf{u}_{1}+\mathbf{F}_{\mathbf{x} \mu}^{0} \mathbf{Z}_{11}+\mathbf{F}_{\mathbf{x x} \mu}^{0} \mathbf{u}_{1}^{2}\right) \widehat{\boldsymbol{\mu}}, \\
\mathbf{C}_{j \mu \mu} \widehat{\boldsymbol{\mu}}^{2}= & \mathbf{v}_{j}^{H}\left(-\mathbf{C}_{j \mu} \mathbf{Z}_{j \mu}+\mathbf{F}_{\mathbf{x} \mu}^{0} \mathbf{Z}_{1 \mu}+\mathbf{F}_{\mathbf{x} \mu \mu}^{0} \mathbf{u}_{1}\right) \widehat{\boldsymbol{\mu}}^{2} \quad(j=1,2) .
\end{aligned}
$$

\section{Appendix B}

The coefficients $C_{i j h}$ and the row vectors $\mathbf{C}_{i j \mu}$ appearing in Equation (22) are given by

$$
\begin{aligned}
& C_{11 \overline{1}}=\mathbf{v}_{1}^{H}\left(\mathbf{F}_{\mathbf{x x}}^{0} \mathbf{z}_{1 \overline{1}} \mathbf{u}_{1}+\mathbf{F}_{\mathbf{x x}}^{0} \overline{\mathbf{z}}_{1 \overline{1}} \mathbf{u}_{1}+\mathbf{F}_{\mathbf{x x}}^{0} \mathbf{z}_{11} \overline{\mathbf{u}}_{1}+\mathbf{F}_{\mathbf{x x x}}^{0} \mathbf{u}_{1}^{2} \overline{\mathbf{u}}_{1}\right), \\
& C_{12 \overline{2}}=\mathbf{v}_{1}^{H}\left(\mathbf{F}_{\mathbf{x x}}^{0} \mathbf{z}_{2 \overline{2}} \overline{\mathbf{u}}_{1}+\mathbf{F}_{\mathbf{x x}}^{0} \overline{\mathbf{z}}_{2 \overline{2}} \mathbf{u}_{1}+\mathbf{F}_{\mathbf{x x}}^{0} \mathbf{z}_{\overline{1} 2} \mathbf{u}_{2}+\mathbf{F}_{\mathbf{x x}}^{0} \mathbf{z}_{12} \overline{\mathbf{u}}_{2}+2 \mathbf{F}_{\mathbf{x x x}}^{0} \mathbf{u}_{1} \mathbf{u}_{2} \overline{\mathbf{u}}_{2}\right), \\
& C_{\overline{1} \overline{1} 2}=\mathbf{v}_{1}^{H}\left(\mathbf{F}_{\mathbf{x x}}^{0} \overline{\mathbf{z}}_{1 \overline{2}} \overline{\mathbf{u}}_{1}+\mathbf{F}_{\mathbf{x x}}^{0} \overline{\mathbf{z}}_{11} \mathbf{u}_{1}+\mathbf{F}_{\mathbf{x x x}}^{0} \overline{\mathbf{u}}_{1}^{2} \mathbf{u}_{1}\right), \\
& C_{111}=\mathbf{v}_{2}^{H}\left(\mathbf{F}_{\mathbf{x x}}^{0} \mathbf{z}_{11} \mathbf{u}_{1}+1 / 3 \mathbf{F}_{\mathbf{x x x}}^{0} \mathbf{u}_{1}^{3}\right), \\
& C_{1 \overline{1} 2}=\mathbf{v}_{2}^{H}\left(\mathbf{F}_{\mathbf{x x}}^{0} \overline{\mathbf{z}}_{\overline{1} 2} \mathbf{u}_{1}+\mathbf{F}_{\mathbf{x x}}^{0} \mathbf{z}_{12} \overline{\mathbf{u}}_{1}+\mathbf{F}_{\mathbf{x x}}^{0} \mathbf{z}_{1 \overline{1}} \mathbf{u}_{2}+\mathbf{F}_{\mathbf{x x}}^{0} \overline{\mathbf{z}}_{1 \overline{1}} \mathbf{u}_{2}+2 \mathbf{F}_{\mathbf{x x x}}^{0} \mathbf{u}_{1} \overline{\mathbf{u}}_{1} \mathbf{u}_{2}\right), \\
& C_{22 \overline{2}}=\mathbf{v}_{2}^{H}\left(\mathbf{F}_{\mathbf{x x}}^{0} \mathbf{z}_{2 \overline{2}} \overline{\mathbf{u}}_{2}+\mathbf{F}_{\mathbf{x x}}^{0} \overline{\mathbf{z}}_{2 \overline{2}} \mathbf{u}_{2}+\mathbf{F}_{\mathbf{x x}}^{0} \mathbf{z}_{22} \overline{\mathbf{u}}_{2}+\mathbf{F}_{\mathbf{x x x}}^{0} \mathbf{u}_{2}^{2} \overline{\mathbf{u}}_{2}\right), \\
& \mathbf{C}_{j \mu} \widehat{\boldsymbol{\mu}}=\mathbf{v}_{j}^{H} \mathbf{F}_{\mathbf{x} \mu}^{0} \mathbf{u}_{j} \widehat{\boldsymbol{\mu}} \quad(j=1,2) .
\end{aligned}
$$

\section{Appendix C}

The linearized bifurcation equations (17) or (22) are of the following type:

$$
\begin{aligned}
& \dot{A}_{1}=c_{1} A_{1} \\
& \dot{A}_{2}=c_{2} A_{2}
\end{aligned}
$$

and admit the eigenvalues $\lambda_{1,2}=c_{1,2}$. Therefore stability of the trivial solution is governed by $\operatorname{Re}\left(c_{1}\right)$ and $\operatorname{Re}\left(c_{2}\right)$. By using the mixed representation (18) or (24), Equations (34) lead to

$$
\begin{aligned}
& \dot{a}_{1}=c_{1 R} a_{1}, \\
& a_{1} \dot{\theta}=c_{1 I} a_{1}, \\
& \dot{u}-\beta v \dot{\theta}=c_{2 R} u-c_{2 I} v, \\
& \dot{v}+\beta u \dot{\theta}=c_{2 R} v+c_{2 I} u,
\end{aligned}
$$

with $\beta=2,3$ and $c_{i R}, c_{i I}(i=1,2)$ the real and imaginary parts of $c_{i}$. By dividing Equation $\left(35_{2}\right)$ by $a_{1}$ and substituting it in Equation $\left(35_{3,4}\right)$, the following linear system is obtained:

$$
\left(\begin{array}{l}
\dot{a}_{1} \\
\dot{u} \\
\dot{v}
\end{array}\right)=\left[\begin{array}{c|cc}
c_{1 R} & & \\
\hline & c_{2 R} & -c_{2 I}+3 c_{1 I} \\
& c_{2 I}-3 c_{1 I} & c_{2 R}
\end{array}\right]\left(\begin{array}{l}
a_{1} \\
u \\
v
\end{array}\right),
$$


admitting the following eigenvalues:

$$
\begin{aligned}
\lambda_{1} & =c_{1 R}, \\
\lambda_{2,3} & =c_{2 R} \pm i\left(c_{2 I}-3 c_{1 I}\right) .
\end{aligned}
$$

Since their real parts are identical to those of Equations (34), it results that division by $a_{1}$ of Equation $\left(35_{2}\right)$ does not alter the stability properties of the trivial solution.

\section{Appendix D}

The dimensional equations of motion of the system in Figure 1a read:

$$
\begin{aligned}
\frac{1}{3} m l^{3} \ddot{q}_{1}= & c_{e} l^{2} \dot{q}_{2}-\left[c_{t}+c_{e} l^{2}+\frac{1}{6} b\left(c_{d}+c_{l}^{\prime}\right) \rho l^{3} U\right] \dot{q}_{1}-k_{t_{1}} q_{1} \\
& +k_{e}^{(1)} l^{2}\left(q_{2}-q_{1}\right)+\frac{1}{2} k_{e}^{(2)} l^{3}\left(q_{2}-q_{1}\right)^{2}+\frac{1}{6} k_{e}^{(3)} l^{4}\left(q_{2}-q_{1}\right)^{3} \\
& +\frac{1}{2} k_{e}^{(1)} l^{2} q_{1}^{2} q_{2}+\frac{1}{6} k_{e}^{(1)} l^{2} q_{2}^{3}+c_{e} l^{2}\left(q_{1}^{2} \dot{q}_{1}-\frac{1}{2} q_{1}^{2} \dot{q}_{2}-\frac{1}{2} q_{2}^{2} \dot{q}_{2}\right) \\
& +\frac{1}{2} \rho b\left[\frac{1}{8}\left(2 c_{d}^{\prime}+c_{l}+c_{l}^{\prime \prime}\right) l^{4} \dot{q}_{1}^{2}+\frac{1}{30 U}\left(3 c_{d}+3 c_{d}^{\prime \prime}+c_{l}^{\prime}+c_{l}^{\prime \prime}\right) l^{5} \dot{q}_{1}^{3}\right], \\
\frac{1}{3} m l^{3} \ddot{q}_{2}= & c_{e} l^{2} \dot{q}_{1}-\left[c_{t}+c_{e} l^{2}+\frac{1}{6} b\left(c_{d}+c_{l}^{\prime}\right) \rho l^{3} U\right] \dot{q}_{2}-k_{t_{2}} q_{2} \\
& -k_{e}^{(1)} l^{2}\left(q_{2}-q_{1}\right)-\frac{1}{2} k_{e}^{(2)} l^{3}\left(q_{2}-q_{1}\right)^{2}-\frac{1}{6} k_{e}^{(3)} l^{4}\left(q_{2}-q_{1}\right)^{3} \\
& -\frac{2}{3} k_{e}^{(1)} l^{2} q_{2}^{3}+c_{e} l^{2}\left(q_{2}^{2} \dot{q}_{2}-\frac{1}{2} q_{1}^{2} \dot{q}_{1}-\frac{1}{2} q_{2}^{2} \dot{q}_{1}\right) \\
& +\frac{1}{2} \rho b\left[\frac{1}{8}\left(2 c_{d}^{\prime}+c_{l}+c_{l}^{\prime \prime}\right) l^{4} \dot{q}_{2}^{2}+\frac{1}{30 U}\left(3 c_{d}+3 c_{d}^{\prime \prime}+c_{l}^{\prime}+c_{l}^{\prime \prime}\right) l^{5} \dot{q}_{2}^{3}\right],
\end{aligned}
$$

where $k_{e}^{(1)}$ is the linear stiffness of the extensional device, $k_{e}^{(2)}$ and $k_{e}^{(3)}$ the quadratic and cubic stiffness (i.e. $f=k_{e}^{(1)} e+k_{e}^{(2)} \mathrm{e}^{2} / 2+k_{e}^{(3)} \mathrm{e}^{2} / 6$ ); $\rho$ is the air density; $b$ is a characteristic length of the cross-section of the bars; $m$ is the mass per length unit of the bars; $c_{d}$ and $c_{l}$ are the nondimensional drag and lift coefficients, respectively, and their apices denote differentiations with respect to the attack angle; $k_{t_{1}}$ and $k_{t_{2}}$ are the linear torsional stiffness; $c_{e}$ and $c_{t}$ are the damping coefficients of the extensional and torsional devices. Equations (38) are nondimensionalized by introducing the following quantities:

$$
\begin{aligned}
& \tau=\omega_{1} t ; \quad \omega_{1}^{2}=\frac{3 k_{t 1}}{m l^{3}} ; \quad \xi_{e}=\frac{3 c_{e}}{2 m \omega_{1} l} ; \quad \xi_{t}=\frac{3 c_{t}}{2 m \omega_{1} l^{3}} ; \quad \alpha_{2}=\frac{3 k_{e}^{(2)}}{2 m \omega_{1}^{2}} ; \quad \alpha_{3}=\frac{3 k_{e}^{(3)}}{2 m \omega_{1}^{2}} ; \\
& c_{1}=\frac{1}{2}\left(c_{d}+c_{l}^{\prime}\right) ; \quad c_{2}=\frac{3}{16} \frac{\rho b l}{m}\left(2 c_{d}^{\prime}+c_{l}+c_{l}^{\prime \prime}\right) ; \\
& c_{3}=-\frac{1}{20}\left(\frac{\rho b l}{m}\right)^{2}\left(3 c_{d}+3 c_{d}^{\prime \prime}+c_{l}^{\prime}+c_{l}^{\prime \prime \prime}\right)
\end{aligned}
$$




$$
\mu=\frac{\rho b}{m \omega_{1}} U ; \quad v=\frac{k_{e}^{(1)}}{m \omega_{1}^{2} l} ; \quad \eta=\frac{k_{t_{1}}}{k_{t_{2}}}
$$

and Equations (25) are obtained. The critical values of the control parameters are the following:

$$
\mu_{0}=-\frac{2\left(\xi_{t}+\xi_{e}\right)}{c_{1}}, \quad v_{0}=0,
$$

for which a double Hopf bifurcation takes place; moreover, at $\eta=\eta_{0}$, with

$$
\eta_{0}=\left\{\begin{array}{c}
\frac{1}{9}\left(41+36 \xi_{e}^{2}+20 \sqrt{\left(4+9 \xi_{e}^{2}\right)}\right), \\
\frac{1}{8}\left(17+32 \xi_{e}^{2}+5 \sqrt{9+64 \xi_{e}^{2}}\right),
\end{array}\right.
$$

the critical eigenvalues are in 1:2 or 1:3 ratio respectively (note that if $\xi_{e} \ll 1, \eta_{0}=\beta^{2}+$ $O\left(\xi_{e}^{2}\right)$, with $\left.\beta=2,3\right)$.

The Jacobian matrix $\mathbf{A}$ and nonlinear vector terms $\mathbf{b}(\mathbf{x})$ in Equations (25) are respectively given by

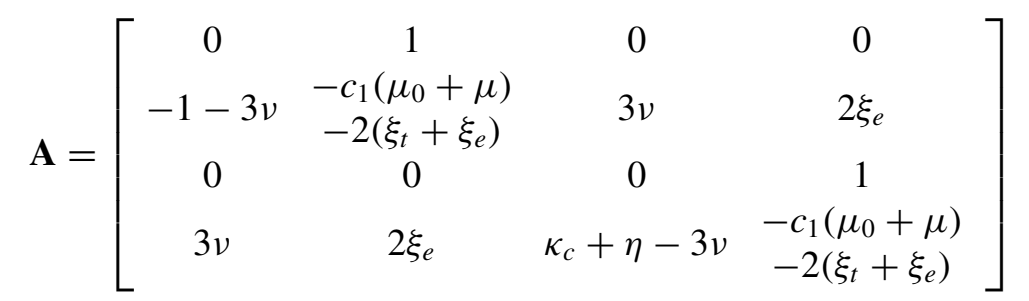

and

$$
\begin{aligned}
b_{1}= & c_{2} x_{2}^{2}+2 v x_{1}^{3}+\frac{c_{3}}{\mu_{0}+\mu} x_{2}^{3}-\frac{3}{2} v x_{1}^{2} x_{3}-\frac{1}{2} v x_{3}^{3}+2 x_{1}^{2} x_{2} \xi_{e}, \\
& -x_{1}^{2} x_{4} \xi_{e}-x_{3}^{2} x_{4} \xi_{e}-\alpha_{2}\left(x_{3}-x_{1}\right)^{2}-\alpha_{3}\left(x_{3}-x_{1}\right)^{3} \\
b_{2}= & c_{2} x_{4}^{2}+2 v x_{3}^{3}+\frac{c_{3}}{\mu_{0}+\mu} x_{4}^{3}-\frac{3}{2} v x_{1} x_{3}^{2}-\frac{1}{2} v x_{1}^{3} \\
& +2 x_{3}^{2} x_{4} \xi_{e}-x_{1}^{2} x_{2} \xi_{e}-x_{2} x_{3}^{2} \xi_{e}+\alpha_{2}\left(x_{3}-x_{1}\right)^{2}+\alpha_{3}\left(x_{3}-x_{1}\right)^{3} .
\end{aligned}
$$

The following values of the nondimensional parameters have been taken for 1:2 and 1:3 cases: $\xi_{e}=\xi_{t}=0.5 ; c_{1}=-1.345 ; c_{2}=0$ (corresponding to a square cross-section of the bars); $c_{3}=-1.251 ; \alpha_{2}=\alpha_{3}=0$ and $\alpha_{2}=\alpha_{3}=1$ for the $1: 3$ and 1:2 cases, respectively.

\section{Acknowledgement}

The work was supported by MURST 40\% funds 2001.

\section{References}

1. Nayfeh, A. H. and Mook, D. T., Nonlinear Oscillations, Wiley, New York, 1979.

2. Luongo, A. and Paolone, A., 'On the reconstitution problem in the multiple time scale method', Nonlinear Dynamics 14, 1999, 133-156. 
3. Nayfeh, A. H. and Balachandran, B., Applied Nonlinear Dynamics, Wiley, New York, 1995.

4. Pignataro, M., Rizzi, N., and Luongo, A., Bifurcation, Stability and Postcritical Behaviour of Elastic Structures, Elsevier, Amsterdam, 1990 (first Italian edition ESA, Roma, 1983).

5. Luongo, A. and Paolone, A., 'Perturbation methods for bifurcation analysis from multiple nonresonant complex eigenvalues', Nonlinear Dynamics 14, 1997, 193-210.

6. Luongo, A. and Paolone, A., 'Multiple scale analysis for divergence-Hopf bifurcation of imperfect symmetric systems', Journal of Sound and Vibration 218, 1998, 527-539.

7. Luongo, A., Paolone, A., and Di Egidio, A., 'Multiple time scale analysis for bifurcation from a double-zero eigenvalue', ASME, Las Vegas, NV, 1999.

8. Paolone, A., Di Egidio A., and Luongo, A., 'Post-critical analysis of self-excited structures in 1:1 resonance condition', in Proceedings of the XIV Italian Congress of Theoretical and Applied Mechanics, Structural Mechanics Section, Como, Italy, 1999 [in Italian].

9. Luongo, A., 'Eigensolutions sensitivity for nonsymmetric matrices with repeated eigenvalues', AIAA Journal 31, 1993, 1321-1328.

10. Luongo, A., Paolone, A., and Di Egidio, A., 'Sensitivities and linear stability analysis around a double zero eigenvalue', AIAA Journal 38(4), 2000, 702-710.

11. Natsiavas, S., 'Free vibration of two coupled nonlinear oscillators', Nonlinear Dynamics 6, 1994, 69-86.

12. Natsiavas, S., 'Free vibration in a class of self-excited oscillators with 1:3 internal resonance', Nonlinear Dynamics 12, 1997, 109-128.

13. Guckenheimer, J. and Holmes, P. J., Nonlinear Oscillations, Dynamical Systems and Bifurcations of Vector Fields, Springer, New York, 1983.

14. Luongo, A., Di Egidio, A., and Paolone, A., 'On the proper form of the amplitude modulation equations for resonant systems', Nonlinear Dynamics 27(3), 2002, 237-254.

15. Doedel, E. and Kernevez, J. P., 'AUTO: Software for continuation and bifurcations problem in ordinary differential equations', Applied Mathematics Report, California Institute of Technology, 1986.

16. Piccardo, G., 'A methodology for the study of coupled aeroelastic phenomena', Journal of Wind Engineering and Industrial Aerodynamics 48, 1993, 241-252. 